\title{
Design and Analysis of Robust and Adaptive Organizations *
}

\author{
Georgiy M. Levchuk \\ Candra Meirina \\ Yuri N. Levchuk \\ Krishna R. Pattipati \\ David L. Kleinman \\ Dept. of Electrical and Computer Engineering \\ University of Connecticut \\ Storrs, CT 06269-3157
}

\begin{abstract}
The objectives of this paper are to: (i) define and classify the process of strategy and structural adaptation of organizations to mission and environmental changes; (ii) apply our modified design methodology to construct robust and adaptive organizations; and (iii) analyze the effects of changes in mission and organizational parameters on the organization's performance. We analyze the performance of organizations using dynamic metrics, including a measure of congruence of a given organization with respect to an organization optimally matched to a mission, as well as the DM activity and task workload as a function of time.

Our previously developed mission modeling and three-phase organizational design methodology allowed one to overcome the computational complexity and to synthesize an organization via an iterative solution of a sequence of smaller and well-defined optimization problems [G. Levchuk et al., 2000a\&b]. This paper extends this methodology to design robust and adaptive organizations.
\end{abstract}

\section{Introduction}

\subsection{Motivation}

The operational and cognitive capabilities of human decision-makers within an organization are limited, which requires that the responsibilities and the ensuing information, resources, and activities be distributed among them with care. In a highly competitive and distributed environment, a proper organizational design that defines the structure and processes is critical to superior organizational performance.

When designing organizations to operate in an uncertain military environment, the specific information about many mission parameters may be inaccessible a priori, with only estimates (e.g., the forms of probability density functions, expected values and standard deviations, etc.) available to the designer. Once the mission scenario unfolds, the actual values of the parameters may require the implementation of a particular strategy to achieve desirable performance.

\footnotetext{
* This work was supported by the Office of Naval Research under contract \# N00014-93-1-0793, \# N00014-98-1-0465 and \# N00014-00-1-0101.
} 
Furthermore, throughout the course of the mission, various causes (e.g., an erroneous initial mission parameter estimation, operational resource failures, malfunctioning of a decision node, etc.) may trigger unexpected changes in either the mission environment or in organizational constraints. Consequently, an on-line structural reconfiguration and/or decision strategy adaptation could become necessary for the successful completion of a mission.

Contingency theorists argue [Burton and Obel, 1998], and the empirical studies concur [Entin, 1999], that the effectiveness of an organization is influenced by the "degree of fit" between the requirements of the environment and the characteristics of an organization. The effort to achieve dynamic congruency in the face of changing environments forces organizations to adapt while they continue to operate [Mackenzie et al., 1996]. Not only an organization must evaluate its design against the environment to know when to adapt, but also, for an organization to succeed, the adaptation process must be smooth, efficient, and cost-effective.

Thus, the challenge is to construct organizational architectures capable of: (i) operating in a timepressured and uncertain mission environment, (ii) capturing the necessary information about unforeseen dynamic changes in a mission environment and/or organization, and (iii) implementing the required on-line adaptation of structure and strategy. For this purpose, the existing scientific models of organizational design (e.g., [G. Levchuk et al., 2000a\&b]) must be enhanced to equip an organization with mission monitoring and structural adaptation schemes. Of paramount importance are: (1) a comprehensive model of both the adaptive organization and its mission, essential for understanding the implications of different adaptation paths; and (2) algorithms that, in the event of adaptation triggers (e.g., changes in the mission environment, operator overload, resource failures, etc.), would allow for rapid search of near-optimal adaptation options.

\subsection{Robust and Adaptive Organizations}

Various strategies may be utilized to build an organization that is commensurate with the dynamic nature of its environment. At one extreme, one may construct an organization capable of processing a range of expected missions. At the other extreme, one may build a 'finely-tuned' organization for a specific mission, and allow online structural reconfiguration and/or strategy adaptation to cope with unforeseen changes in the mission and/or an organization. The former (multi-mission) organizations, herein termed robust, are able to sustain high levels of performance in dynamic environments without having to change their structures. The latter organizations, herein termed adaptive, are able to generate new strategies and/or reconfigure their structure to potentially achieve even higher performance.

\subsubsection{Robust Organizations}

Robust organizations can maintain an acceptable performance in a changing environment without having to change organizational structure. This is usually achieved by introducing redundancies in task-resource allocation, which makes organizations more stable with respect to environmental perturbations and/or decision/processing errors. In order to achieve robustness, an organization is designed to process a range of missions. Evidently, this insensitivity results in slightly degraded performance on each specific mission, but minimizes the organization's 
fragility. In this paper, we consider the problem of designing robust organizations taking into account potential contingencies that may occur in a mission.

\subsubsection{Adaptive Organizations}

The dynamic and uncertain nature of a mission faced by a distributed multi-DM organization brings new requirements to cost-effective organizational design and operations. In an uncertain environment, it may be more cost-efficient for an organization simply to alter its structure and processes to environmental changes than to waste resources to preserve the redundancy required to cope with many possible mission outcomes. Adaptive organizations are capable of modifying processing strategies and structure to maintain high performance.

Flexibility in design is the key to success of adaptive organizations. We consider the following types of organizational flexibility (following [Tsourveloudis and Phillis, 1998]):

1) Processing flexibility (task assignment, task-resource allocation, and task processing sequencing). This type of flexibility measures the ability of an organization to deal with changes in task processing.

2) Planning flexibility allows for quick reaction to unexpected events such as processing node/resource failures, and minimizes the effect of task interruptions on a mission schedule. This type of flexibility is related to operational commonality, i.e., the number of common task-resource and DM-resource pairings that the organization can utilize; and substitutability, namely the ability to replan and reschedule tasks by employing equivalent resource packages under failure conditions. Both planning and processing flexibility are interdependent and influence the strategy adaptation employed by an organization.

3) Resource allocation flexibility measures the ability of an organization to reassign its resources in response to changes in a task environment; and

4) Hierarchy flexibility pertains to the ability to shift DM-DM coordination/authority structure. Both resource allocation and hierarchy flexibility are interdependent and determine the structural adaptation procedures used by an organization.

In order to maintain its mission schedule in a time-pressured environment, an adaptive organization must be able, in a timely fashion, to capture/analyze the necessary information, examine its adaptation alternatives, and implement the right adaptation option. For an organization to be able to adapt and still maintain its mission schedule, the adaptation phase must be compatible with the processing of mission tasks (in some cases, the adaptation time must be significantly smaller than the time to process the corresponding mission tasks to allow for successful completion of the mission). In order to determine if adaptation is required, the appropriate mission/organization monitoring data should be analyzed, and failure diagnostics should be performed in a timely fashion.

Organizational adaptation process is significantly simplified if specific causes for adaptation, or adaptation triggers, are anticipated a priori. These are the unexpected changes in the mission environment, resource failures, DM failures, etc., that require an organization to adapt (that is, the organization can no longer sustain a high level of performance without changing its structure and/or strategy). After a suitable adaptation option (e.g., strategy shift, resource reallocation, hierarchy reconfiguration) is selected, the organization needs to coordinate among its members to realize the selected change. In hierarchical organizations, the authority to declare and enforce on-line reconfiguration belongs to the superior DM (known as the root DM). Hence, it is natural 
(although not necessary) that a decision to adapt be taken at this highest level of the command hierarchy.

\subsection{Organizational congruence}

Mackenzie [Mackenzie, 1986] identifies three main interdependencies in complex organizations:

- Task Process interdependence - a precedence structure among tasks to be processed;

- Task-Resource interdependence - task-resource characteristics (such as location, personnel, technology, timing, knowledge, information), continuity of direction, coordination and control, and resource flows for task processing; and

- Environmental interdependence - a combination of interdependencies of the organization's task processes, task-resource characteristics, and positions created and affected by the organization's environment.

The main reason for analyzing organizational interdependencies is to determine which are necessary, to structure them so that they intertwine, and to eliminate excess interdependencies. Excess interdependencies create waste and confusion, inhibit initiative, and generate disputes among senior management personnel. There exists a proper level of interdependency in an organization to operate in a manner consistent with the attainment of its goals.

The best way to handle environmental interdependencies is to first reduce the intraorganizational interdependencies (Task Process and Task-Resource interdependencies) and then to seek organizational congruence with the mission at hand. The organization is said to be congruent with its mission if its structure and processes are matched to the environmental parameters. Note that simply building the organization to optimize an objective function constructed from mission objectives does not necessarily result in a congruent organization. For example, if the goals of an organization are based on some erroneous assumptions, then they do not match the mission environment, and, consequently, result in unacceptable performance. Another example involves goals that are not consistent with the technology at hand, thereby making the functioning of an organization infeasible. Mackenzie [Mackenzie, 1986] presents eleven necessary conditions for an organization to be congruent:

1. Goals and environments are congruent.

2. Strategies and environments are congruent.

3. Goals and strategies are congruent.

4. Senior management agrees on goals, strategies, and environments.

5. Environmental design premises and the environments are congruent.

6. Strategic design premises and the strategies are congruent.

7. Organizational design premises and the organizational logic are congruent.

8. Organizational logic and the organizational architecture are congruent.

9. Organizational architecture and the actual organization are congruent.

10. Results are congruent with the organizational technology.

11. Results are congruent with the goals.

Evidently, this is a general and vague definition. To make it more precise, we note that an organization that is designed to optimize an objective function, constructed from the mission goals consistent with the environment, would have the best feasible performance. Therefore, it would be the most congruent one with the mission among all possible organizations operating 
under the same environmental design premises and logic (that is, the match between its parameters and the mission would be close). Hence, comparing any other organizations' performance to this "best" organization would indicate the degree of their congruence to the mission. This idea of performance-based congruence could be extended to include the match between structures and processes as well.

How precise or loose should the congruence be? A loose match between organizational and mission structures may be better if it is robust to changes in the mission (although possibly at the expense of performance degradation and additional personnel cost). An organizational structure that is precisely matched to a mission may exhibit brittle behavior in an uncertain environment. Experiments have shown that congruence between resource allocation structure and mission structure improves organizational performance (e.g., see [Entin, 1999], [Hocevar et al., 1999]). A substantial decrease in the degree of congruence can signal the need for dynamic reconfiguration.

\subsection{Related Research}

The robust design approach originated in quality planning and engineering product design activities [Taguchi, 1986 and 1987]. Taguchi states that it is often more costly to control causes of process variation than to make the system insensitive to the dynamics. In [Villas Boas, 1999] Taguchi's robust engineering, from its early introduction in the fifties through the present day, is reviewed. In her recent work, Sanchez [Sanchez, 2000] shows that the use of a loss function, which incorporates both the system mean and system variability, enables system optimization and improvement efforts to proceed efficiently and effectively. Other recent work in this area maybe found in [Song et al., 1995], [Pachucki, 1995], [Chunming, 1995], [Kalyanaram, 1997], [Mathur and Pattipati, 1997], and [Scibilia et al., 1999].

Equally attractive is the approach to construct adaptive organizations. This is motivated by a tendency of robust design approach to overly-engineer each aspect of a system to reduce the chances of failure. Typically, it increases the system overhead and decreases the level of system efficiency, since the projected variations may not occur in practice. In order to improve the ability of an organization to accommodate variations during the course of its mission, without sacrificing its efficiency, organizations should be designed with adaptability in mind.

In [Foisel et al., 1998], a model is introduced to build a multi-agent system with global coherent behavior through the use of an organizational structure that can adapt in a distributed fashion. They employ interaction models to provide an initial organizational structure and utilize local assessment capabilities to make agents adapt their structure in response to their local requirements. An organizational adaptation via dynamic process selection is presented in [Handley et al., 1999]. A virtual experiment was conducted to simulate the performance of an organization with local and global adaptation strategies. The timeliness of organization's response was used as a performance measure. When changes occur, either within the organization or in its environment, to such an extent that the organization is no longer performing adequately or cannot perform in its current configuration, the organization reconfigures itself in response to these changes. In [Lee and Carley, 1997], tuning and shaking are proposed as meta-strategies for organizational adaptation. Their work is based on simulated annealing, and the adaptation strategy is modeled as having two components of the annealing process. Tuning occurs when the organization changes its structure at a decreasing rate over time, whereas a shake-up process occurs when the organization increases the likelihood of 
making major changes in the structure. Carley [Carley, 1998] views organizations as computational entities, and argues that adaptation insights may be gained by examining such processes in an organization of artificial agents. The results suggest that both organizational performance and form depend on environmental change, agent-learning process, and the emergence of institutionalized strategies. The importance of capturing the knowledge of downstream organizational changes is emphasized in [Heller, 2000]. It is noted that a model in which informational knowledge of what new activities are required to implement a new product must be complemented with interpretive knowledge of how those activities relate to the organization and strategy of the firm. Other research in adaptation includes [Y. Levchuk et al., 1998], [Dill and Pearson, 1991], and [Horling et al., 1999].

Despite many advantages that an adaptive organization offers, it has its own shortcomings. In the context of agile manufacturing systems, Booth [Booth, 1996] argues that 'lean' production concepts have put the companies at the risk of not being able to recover from unforeseen situations due to reductions in skilled staff in general, and in design and development capability in particular. To become agile, companies have to aim for flexibility, speed of response, and adaptability to probable changes. The underlying criticism highlights the fact that there is a compromise, and possibly a better approach, in combining the two equally promising design procedures, viz., robustness and flexibility. The notion of organizational adaptability points to the need for an organization to be both robust to accommodate small variations in the system and flexible enough to maintain a high level of performance by adapting strategies and structures to accommodate the effects of large changes during the course of a mission. Other work on flexible system design may be found in [Piramuthu et al., 1994], [Kulatilaka, 1988], and [Peddie et al., $1991]$.

In the context of organizational design, it has long been accepted that congruency between a mission and the concomitant organizational structure and processes is necessary to achieve superior performance [Mackenzie, 1986]. A key prerequisite to a superior organizational design is the availability of effective and insightful performance measure(s). In general, there is little consensus on what constitutes organizational performance, and there is no universally best performance measure(s). As was shown in [Cameron, 1986], whether an organization is said to perform well depends on the constraints placed on the performance measures and on organizational objectives. Organizational performance can be viewed from a variety of perspectives, such as productivity [Argote and Epple, 1990], profitability [Lawrence and Lorsch, 1967], reliability [Roberts, 1989], timeliness [Handley et al., 1999], accuracy [Lee and Carley, 1997], and aggregation of expected cost, quality, and duration [Horling et al., 1999].

The challenge is to develop effective performance measures and use them in evaluating an organization's performance and further utilize them in building proper organizations depending on the missions. In [Bucheim, 2000], the need for constructing effective performance metrics to improve competitiveness, to high-light areas needing improvement, to help in focusing design emphasis on the customer's desires and priorities, and to build teamwork between engineering and the other functions of a corporation was illustrated. Lefebvre [Levebvre et al., 1997] investigates the relationship between organizational fit and performance, specifically the relationship between the level of internal coherence and the firm's performance.

\subsection{Organization of the Paper}


This paper extends our design methodology to synthesize robust and adaptive organizations. Section 2 contains a definition of organizational design problem, describes our 3-phase organizational design process, and introduces the performance and congruence measures to be utilized in assessing the performance of organizations. Section 3 presents the application of our design methodology to construct robust organizations. In section 3.3, we describe our methodology to design robust organizations via concatenation of a range of expected missions. An example of robust design is given in section 3.4. Application of our methodology to structural adaptation is presented in section 4. In this section, we define the cost of structural adaptation, present an algorithm to devise the adaptation path, and conclude with an example of structural adaptation. Summary and future extensions are discussed in section 5.

\section{Organizational Design Process}

\subsection{Mission Environment and Organizational Design Problem}

A complex joint-operations mission is defined by specifying a set of inter-dependent tasks to be performed in order to achieve the mission objectives. The interrelationships among the tasks (i.e., precedence, data flow, etc.) can be represented via dependency diagrams, i.e., a directed acyclic graph represents a plan to execute a mission. A task is an activity that entails the use of relevant resources and is carried out by an individual decision-maker (DM) or a group of DMs to accomplish the mission objectives. Every task in itself represents a small mission and oftentimes can be further decomposed into more elementary tasks. A mission decomposition diagram can be built to represent the hierarchical structure among the mission tasks [G. Levchuk, 2000a]. Various decomposition techniques (e.g., goal decomposition, domain decomposition, functional decomposition) represent different starting points for defining tasks and provide different task types required to complete a mission. The designer's choice of a particular decomposition technique and model granularity (number of tasks in the mission decomposition) must be contingent upon the desired degree of model fidelity and the computational efficiency of the design process and of its supporting algorithms.

An organization can be viewed as a system composed of individual decision-makers together with organizational resources (processing elements/capabilities, such as platforms, information acquisition/processing units, etc.). Similarly to a mission, the structure of an organization can be modeled as another dependency diagram representing individual DMs and their command hierarchy, as well as DMs' platform/resource ownership. An allocation of platforms to DMs specifies the operational capabilities of DMs ("who can do what"). A hierarchy (an interdependence structure connecting the components of an organization) is created in order to accomplish a set of tasks that individual decision-makers cannot perform alone. It is structured to regulate the inter-DM coordination (to support DM interactions and to resolve the decision ambiguities among coordinating DMs) and to specify the control responsibilities of DMs. An organizational hierarchy can be modeled by defining a partial ordering over a set of DMs (i.e., a directed tree structure over DM nodes) and specifying the coordination rules and DM control responsibilities.

The optimal organizational design problem is one of finding both the optimal organizational structure (e.g., decision hierarchy, allocation of resources and functions to decision-makers 
(DMs), communication structure, etc.) and strategy (allocation of tasks to DMs, sequence of task execution, etc.) that allow the organization to achieve desired performance during the conduct of a specific mission [Y. Levchuk et al., 1998]. Mission objectives together with the design parameters affecting organizational performance form an objective function that serves as an optimization criterion for organizational design.

\subsection{Overview of the 3-Phase Organizational Design Process}

Our previously developed mission modeling and three-phase organizational design methodology allowed one to overcome the computational complexity via an iterative solution of a sequence of smaller and well-defined optimization problems [Y. Levchuk et al., 1997] in synthesizing a command structure. Mixing and matching different optimization algorithms at different stages of the design process [G. Levchuk et al., 2000a\&b] leads to an efficient matching between the mission structure and that of an organization and its resources/constraints.

The notion of optimality is subjective [Keeney and Raiffa, 1993]. Moreover, different aspects of organizational performance are deemed important as the efficacy of an organization is assessed. Hence, the organizational design problem is inherently multi-objective, and the correct choice of optimization criteria is critical to generating the optimal design. The relative weights of the optimization criteria that determine organizational performance can be represented via weighting coefficients assigned to each component in the objective function. Therefore, in theory, we can build an organizational structure by iteratively optimizing different structural dimensions, beginning with those dimensions that delineate the heaviest portion of the objective function. For example, an organizational strategy determines the mission processing schedule as well as the individual operational workload of a DM. Consequently, it generally specifies a large portion of parameters in the multi-variable objective function. Each subsequent dimension is optimized subject to a fixed structure on those dimensions that have been optimized already. The iterative application of optimization process allows one to simultaneously optimize multiple dimensions [Y. Levchuk et al., 1997].

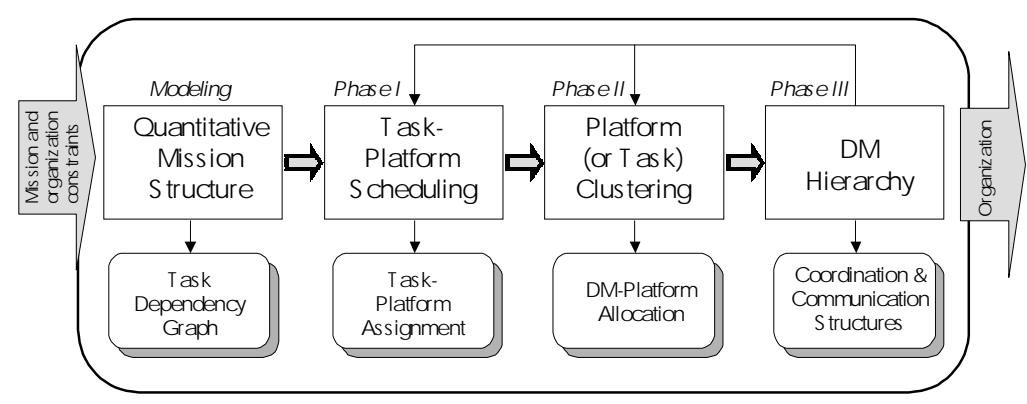

Figure 1: Three Phase Organizational Design Process

Following the above logic, our organizational design methodology integrates various algorithms that optimize different dimensions of an organizational structure. For a given mission structure, an organization is designed via the following three phases (see Fig.1).

For detailed description of algorithms used at each phase of organizational design process, see [G. Levchuk et al., 2000a\&b]. 


\subsection{Mathematical Problem Formulation and Solution Approach}

\subsubsection{Definitions}

A. Tasks

In our model, we characterize each mission task $T_{i}$ by specifying the following basic attributes:

- Estimated processing time $t_{i}(i=1, \ldots, N$, where $N$ is the number of tasks);

- Geographical locations $\left(\boldsymbol{x}_{i}, \boldsymbol{y}_{i}\right)$ (that specifies the concomitant "distance" $d_{i j}$ to be traveled between tasks $T_{i}$ and $T_{j}$ );

- Resource requirement vector $\left[R_{i 1}, R_{i 2}, \ldots, R_{i L}\right]$, where $R_{i l}$ is the number of units of resource $l$ required for successful processing of task $T_{i}(l=1, \ldots, L$, where $L$ is the number of resource types);

- Precedence constraints: $p_{i j}=\left\{\begin{array}{l}0, \text { if task } T_{i} \text { must be completed before task } T_{j} \text { can start } \\ 1, \text { otherwise }\end{array}\right.$

- Information flow $f_{i j}$ - amount of information necessary for processing of task $T_{j}$ related to its predecessor task $T_{i}$ (this information is viewed as units of time needed for information transfer; such a transfer is only necessary if these tasks are assigned to different DMs); the notion of information flow can be viewed as a time-delay in processing task $T_{j}$ caused by assignment of task $T_{i}$ to a different DM.

\section{B. Platforms}

A Platform is a physical asset of an organization that provides resource capabilities and is used to process tasks. For each platform $P_{m}(m=1, \ldots, R)$ we define its maximal velocity $v_{m}$ and its resource capability vector $\left[r_{m 1}, r_{m 2}, \ldots, r_{m L}\right]$, where $r_{m l}$ specifies the number of units of resource type $l$ available on platform $P_{m}$.

\section{Decision-makers (DMs)}

A Decision-maker (DM) is an entity with information-processing, decision-making, and operational capabilities that can control the necessary resources to execute mission tasks, provided that such an execution will not violate the concomitant capability thresholds. A maximal number $D$ of available DMs is specified.

\subsubsection{Phase I (scheduling)}

A set of tasks with specified processing times, resource requirements, locations, and precedence relations must be executed by a set of platforms with given resource capabilities, ranges of 
operation, and velocities. Tasks are allocated to groups of platforms in such a way that, for each such platform package to task assignment, the vector of task's resource requirements is component-wise less than or equal to the aggregated resource capability of the platform group. The task processing can begin only when the processing of all its predecessors is completed and all platforms from the group assigned to this task have arrived at the appropriate location. In our model, we assume that a platform can only process one task at a time. Platforms are to be routed among the tasks so that the overall Mission Completion Time (i.e., the completion time of the last mission task) is minimized. An output of the scheduling phase specifies a platform-task assignment for our organizational design, delineating task start times and platform-task routing.

\section{A. Algorithms}

○ List scheduling with greedy task selection via

- Critical Path;

- Level Assignment;

- Weighted Length;

- Weighted Critical Path.

and greedy platform selection via

- Min Distance;

- Min Expended Resources;

- Max Process Resources.

○ Pair-wise exchange (neighborhood search).

(For additional details, see [G. Levchuk, 2000a])

\section{B. Outputs}

- Assignment variables:

$$
x_{i m}=\left\{\begin{array}{l}
1, \text { if platform } P_{m} \text { is assigned to task } T_{i} \\
0, \text { otherwise }
\end{array}\right.
$$

- Traversing variables:

$$
w_{i j m}=\left\{\begin{array}{l}
1, \text { if platform } P_{m} \text { is assigned to process task } T_{j} \\
\quad \operatorname{after} \text { processing } \operatorname{task} T_{i} \\
0, \text { otherwise }
\end{array}\right.
$$

○ $s_{i}=$ start time of task $T_{i}$. If precedence variable $p_{i j}=0$ (i.e., task $T_{i}$ must be completed before Task $T_{j}$ ), then start times are related via

$$
s_{j} \geq\left\{\begin{array}{l}
s_{i}+t_{i}+\sum_{m=1}^{R} \frac{d_{i j}}{v_{m}} w_{i j m}, \text { if both tasks } T_{i} \text { and } T_{j} \text { are assigned to } D M_{k} \\
s_{i}+t_{i}+\max \left[f_{i j}, \sum_{m=1}^{R} \frac{d_{i j}}{v_{m}} w_{i j m}\right], \text { otherwise }
\end{array}\right.
$$


where $v_{m}$ is the maximum platform velocity and $R$ is the number of platforms owned by an organization.

○ $Y=$ mission completion time (time when the last task is completed).

\subsubsection{Phase II (clustering)}

The platforms are grouped into disjoint clusters according to their task assignments, and these platform clusters are then allocated to different DMs who inherit the corresponding task assignments. The objective of platform clustering is to minimize the resultant DM workload - a weighted sum of external DM-DM (i.e., inter-DM) coordination and internal platform coordination load of a DM [G. Levchuk, 2000b].

After the scheduling phase, we had: $s_{j} \geq \max \left[\sum_{m=1}^{R}\left(s_{i}+t_{i}+\frac{d_{i j}}{v_{m}}\right) w_{i j m},\left(1-p_{i j}\right)\left(s_{i}+t_{i}\right)\right]$.

When DM-platform allocation is found, we update task start times to account for inter-DM information exchange. We would have:

$$
s_{j} \geq \max _{i}\left[\sum_{m=1}^{R}\left(s_{i}+t_{i}+\frac{d_{i j}}{v_{m}}\right) w_{i j m},\left(1-p_{i j}\right)\left(s_{i}+t_{i}+f_{i j} \cdot \mathbf{1}\left(\sum_{k=1}^{D} u_{k j} \sum_{n=1, n \neq k}^{D} u_{n i}>0\right)\right)\right]
$$

where

$$
\mathbf{1}(A)=\left\{\begin{array}{l}
1, \text { if } A=\text { true } \\
0, \text { otherwise }
\end{array}\right.
$$

\section{A. Algorithms}

- Min-Max Workload;

○ Dynamic Clustering;

- Min Dissimilarity;

- Max Similarity; and

- Best Merge.

(For additional details, see [G. Levchuk, 2000b])

\section{B. Outputs}

$$
\begin{aligned}
y_{k m} & =\left\{\begin{array}{l}
1, \text { if } D M_{k} \text { is allocated to platform } P_{m} \\
0, \text { otherwise }
\end{array}\right. \\
u_{k i} & =\left\{\begin{array}{l}
1, \text { if } D M_{k} \text { is assigned to task } T_{i} \\
0, \text { otherwise }
\end{array}\right. \\
& =\left\{\begin{array}{l}
1, \text { if there exists a platform } P_{m} \text { such that } y_{k m}=1, x_{i m}=1 \\
0, \text { otherwise }
\end{array}\right.
\end{aligned}
$$

\subsubsection{Phase III (hierarchy)}


A hierarchy is a partial order relationship that can be viewed as a tree-type network among DM nodes (with "root" DM being the team leader). Oftentimes, a hierarchy induces a structure for decision cycles and information flows associated with inter-DM coordination in an organization. One of the goals in creating a specific hierarchy is to match the induced superior-subordinate DM relationships with the inter-DM coordination required to complete the mission. We complete the organizational design by specifying: (i) a communication structure, and (ii) a decision hierarchy (a directed tree spanning DM nodes) to optimize the responsibility distribution and inter-DM control coordination, as well as to balance the control workload among DMs according to their expertise constraints. Different optimization objectives for organizational design (e.g., maximizing the speed of command by minimizing average decision cycles in the organization; minimizing the management cost associated with coordination overhead; etc.) prompt different rules for building the hierarchy and for selecting its root DM (team leader).

\section{A. Algorithms}

○ Min-Max;

- Min Coordination Cost; and

- Max Aggregated Coordination.

(For more details, see [G. Levchuk, 2000b])

\section{B. Output}

$$
e_{i j}=\left\{\begin{array}{l}
1, \text { if there is a direct link between } D M_{i} \text { and } D M_{j} \text { in the tree } \\
0, \text { otherwise }
\end{array}\right.
$$

\subsection{Organizational Measures}

In this section, we introduce performance measures to compare organizations and to evaluate their degrees of congruence and robustness. As noted earlier, the performance of an organization is a real measure of congruence, since just constructing an organization to optimize an objective function (composed of organization's goals) does not necessarily result in a congruent one, since any of the congruence conditions (1 through 11) may be violated. For example, if organization's strategies/goals happened to be incongruent with the environment, then the entire organizational design would be based on the wrong initial assumptions; the performance of such an organization could be low.

\subsubsection{Aggregated and Dynamic Measures}

The following measures can be measured or computed during mission processing or after a mission is completed:

\section{A. Aggregated Measures}


- Platform travel time $p t_{m}$ (the total time during the mission a platform $P_{m}$ is directed to travel among tasks): $p t_{m}=\sum_{i, j=1}^{N} w_{i j m} \cdot \frac{d_{i j}}{v_{m}} ;$

- Platform processing time $p p_{m}$ (the total time during the mission a platform $P_{m}$ is used for processing tasks): $p p_{m}=\sum_{i=1}^{N} x_{i m} \cdot t_{i}$;

- Time delay per task $d t_{i}, i=1, \ldots, N$. The delay in task processing is the number of units of time the task's starting time should be shifted to account for task information flow (and the corresponding DM-DM information exchange);

- Time delay per platform $d p_{m}$ (sum of all delays of tasks assigned to platform $P_{m}$ ):

$$
d p_{m}=\sum_{i=1}^{N} x_{i m} \cdot d t_{i} .
$$

- Time delay per DM $d d_{k}$ (sum of all delays of tasks assigned to $D M_{k}$ ):

$$
d d_{k}=\sum_{i=1}^{N} u_{k i} \cdot d t_{i}
$$

\section{B. Dynamic Activity Measures}

- Number of tasks $a t_{k}(t)$ processed by $D M_{k}$ at time $t$ :

$$
a t_{k}(t)=\sum_{i=1}^{N} u_{k i} \cdot \mathbf{1}\left(\left(s_{i} \leq t\right) \cap\left(s_{i}+t_{i}>t\right)\right)
$$

Again, recall that $\mathbf{1}(A)=\left\{\begin{array}{l}1, \text { if } A=\text { true } \\ 0, \text { otherwise }\end{array}\right.$.

\section{Dynamic Coordination Measures}

- Number of tasks $c t_{k}(t)$ over which $D M_{k}$ coordinates with other DMs at time $t$ :

$$
c t_{k}(t)=\sum_{i=1}^{N} u_{k i} \cdot \mathbf{1}\left(\left(s_{i} \leq t\right) \cap\left(s_{i}+t_{i}>t\right) \cap\left(\sum_{n=1, n \neq k}^{D} u_{n i}>0\right)\right)
$$

- Number of DMs $c d_{k}(t)$ to coordinate with $D M_{k}$ at time $t$ :

$$
c d_{k}(t)=\sum_{n=1, n \neq k}^{D} \mathbf{1}\left(\exists i: u_{k i} u_{n i}=1\right)
$$

- Amount (weight) of coordination $c w_{k}(t)$ of $D M_{k}$ at time $t$ :

$$
c w_{k}(t)=\sum_{n=1, n \neq k}^{D} \sum_{i=1}^{N} u_{k i} u_{n i}
$$

- Number of tasks $o t_{k}(t)$ over which the coordination overhead exists at $D M_{k}$;

- Number of DM-DM links $o d_{k}(t)$ (information flow paths) through $D M_{k}$;

- Weight of coordination overhead $o w_{k}(t)$ at $D M_{k}$. 


\section{Dynamic Information Exchange Measures}

- Number of tasks $e t_{k}(t)=e t_{k}^{\text {out }}(t)+e t_{k}^{\text {in }}(t)$ over which $D M_{k}$ exchanges information with other DMs at time $t$ :

$$
\begin{aligned}
& e t_{k}^{\text {out }}(t)=\sum_{i=1}^{N} u_{k i} \mathbf{1}\left(\exists j \neq i: p_{i j}=0, s_{i}+t_{i}<t+f_{i j}, s_{i}+t_{i} \geq t, \sum_{n=1, n \neq k}^{D} u_{n i}>0\right) \\
& e t_{k}^{i n}(t)=\sum_{j=1}^{N} \mathbf{1}\left(\exists i \neq j: u_{k i}=1, p_{j i}=0, s_{j}+t_{j}<t+f_{j i}, s_{j}+t_{j} \geq t, \sum_{n=1, n \neq k}^{D} u_{n j}>0\right)
\end{aligned}
$$

- Number of DMs $e d_{k}(t)=e d_{k}^{\text {out }}(t)+e d_{k}^{\text {in }}(t)$ that $D M_{k}$ has information exchange with at time $t$ :

$$
\begin{aligned}
& e d_{k}^{\text {out }}(t)=\sum_{n=1, n \neq k}^{D} \mathbf{1}\left(\exists i, j(j \neq i): u_{k i} u_{n j}=1, p_{i j}=0, s_{i}+t_{i}<t+f_{i j}, s_{i}+t_{i} \geq t\right) \\
& e d_{k}^{\text {in }}(t)=\sum_{n=1, n \neq k}^{D} \mathbf{1}\left(\exists i, j(j \neq i): u_{k j} u_{n i}=1, p_{j i}=0, s_{j}+t_{j}<t+f_{j i}, s_{j}+t_{j} \geq t\right)
\end{aligned}
$$

- Weight of information exchange $e w_{k}(t)=e w_{k}^{o u t}(t)+e w_{k}^{\text {in }}(t)$ for $D M_{k}$ at time $t$ :

$$
\begin{aligned}
& e w_{k}^{\text {out }}(t)=\sum_{n=1, n \neq k}^{D} \sum_{i=1}^{N} u_{k i} u_{n j}\left(1-p_{i j}\right) \cdot \mathbf{1}\left(s_{i}+t_{i}<t+f_{i j}, s_{i}+t_{i} \geq t\right) \\
& e w_{k}^{i n}(t)=\sum_{n=1, n \neq k}^{D} \sum_{i=1}^{N} u_{k j} u_{n i}\left(1-p_{j i}\right) \cdot \mathbf{1}\left(s_{j}+t_{j}<t+f_{j i}, s_{j}+t_{j} \geq t\right)
\end{aligned}
$$

\subsubsection{Performance Measures}

Each dynamic activity of an organizational element typically corresponds to a staircase function. The changes occur at the time of initiation or termination of a task. The average value is found as the mean of this staircase function (integral of this function over time-span of a mission divided by the mission completion time). We combine these dynamic measures into the following measures of performance of an organization on a specific mission (see Appendix for details):

\section{A. Activity}

- $\quad P R=$ Platform routing efficiency per DM

- $D T=$ Task delay efficiency

- $D P=$ Platform delay efficiency

- $D D=$ DM delay efficiency

- $A T=$ DM-task activity efficiency

\section{B. Coordination}

- $C T=$ DM-task coordination efficiency

- $C D=$ DM-DM coordination efficiency

- $C W=\mathrm{DM}$ coordination workload efficiency

- $O T=$ DM-task overhead efficiency 
- $O D=$ DM-DM overhead efficiency

- $O W=$ DM workload overhead efficiency

\section{Information Exchange}

- $I T=$ DM-task information exchange efficiency

- $I D=$ DM-DM information exchange efficiency

- $I W=$ DM information exchange workload efficiency

\subsubsection{Measures of Congruence}

As stated earlier, one way to evaluate the congruence of an organization to a mission is to compare its performance to that of an organization optimally matched to the mission. We say that an organization is congruent to a mission if its performance is above some specified threshold of performance. In addition, an organization, which is specifically designed to execute a mission by way of optimizing an objective function constructed from organization's objectives, would have the best performance if the mission environment and the objectives were congruent. The measure can be extended to include structural and process (i.e., scheduling and resource allocation) match between two organizations as well. Here we formalize the notion of performance-based congruence measure.

Two of the important performance measures of an organization are related to task processing and DM coordination. They are evaluated correspondingly as vectors $\underline{\Pi}$ and $\underline{\Theta}$ :

$$
\begin{aligned}
& \underline{\Pi}(O, M)=[P R, D T, D P, D D, A T, I T, I D, I W] \\
& \underline{\Theta}(O, M)=[C T, C D, C W, O T, O D, O W]
\end{aligned}
$$

where the measures were defined earlier in subsection 2.4.2. Here we introduce a measure of degree of congruence of an organization $O$ to a mission $\boldsymbol{M}$ (with organization $O^{M}$ being optimal for a mission $\boldsymbol{M}$ ) as a two-dimensional vector:

$$
\underline{\boldsymbol{C}}(O, \boldsymbol{M})=\left[\boldsymbol{c}_{1}, \boldsymbol{c}_{2}\right]=\left[\frac{\left\|\underline{\Pi}\left(O^{M}, \boldsymbol{M}\right)\right\|_{2}}{\|\underline{\Pi}(O, \boldsymbol{M})\|_{2}}, \frac{\left\|\underline{\Theta}\left(O^{M}, \boldsymbol{M}\right)\right\|_{2}}{\|\underline{\Theta}(O, \boldsymbol{M})\|_{2}}\right]
$$

Clearly, $\underline{\boldsymbol{C}}(O, \boldsymbol{M}) \in[0,1] \mathrm{x}[0,1]$, and $\underline{\boldsymbol{C}}\left(O^{M}, \boldsymbol{M}\right)=[1,1]$.

\section{Design of Robust Organizations}

\subsection{Organizational Design Example}

A joint group of Navy and Marine Forces is assigned to complete a military mission (mission $\boldsymbol{M}$ ) that includes capturing a seaport and airport to allow for the introduction of follow-on forces. There are two suitable landing beaches designated "North" and "South", with a road leading from the North Beach to the seaport, and another road leading from the South Beach to the airport. The geographic layout of the mission is shown in Fig. 2. 
From intelligence sources, the approximate concentration of hostile forces is known, and counter-strikes are anticipated. The commander devises a plan for the mission that includes the completion of tasks shown in Fig. 2. The following 8 resource requirements/capabilities are modeled: AAW (Anti-Air Warfare), ASUW (Anti-Surface Warfare), ASW (Anti-Submarine Warfare), GASLT (Ground Assault), FIRE (Artillery), ARM (Armor), MINE (Mine Clearing), DES (Designation). Task parameters are shown in Table I. Mission precedence task graph is shown in Fig. 3. The organization has resources displayed in Table II. It has 5 available decision-makers (DMs).

Map

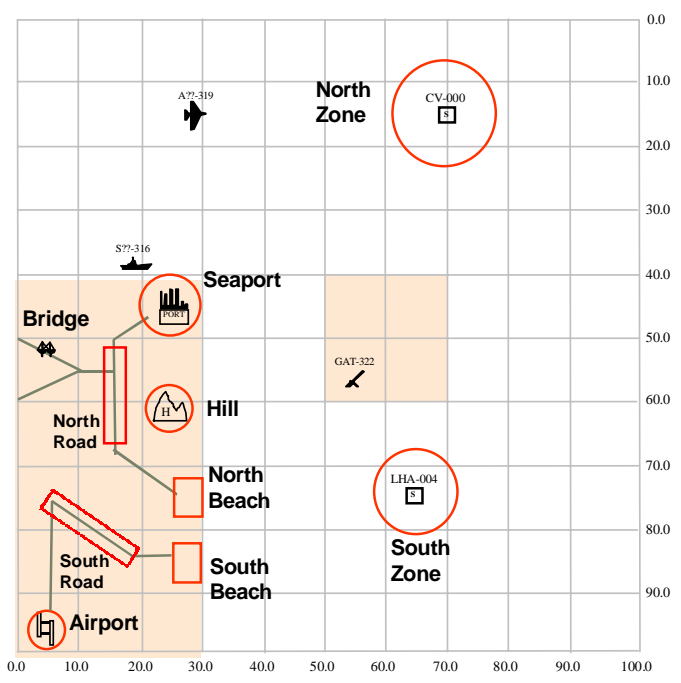

Task List

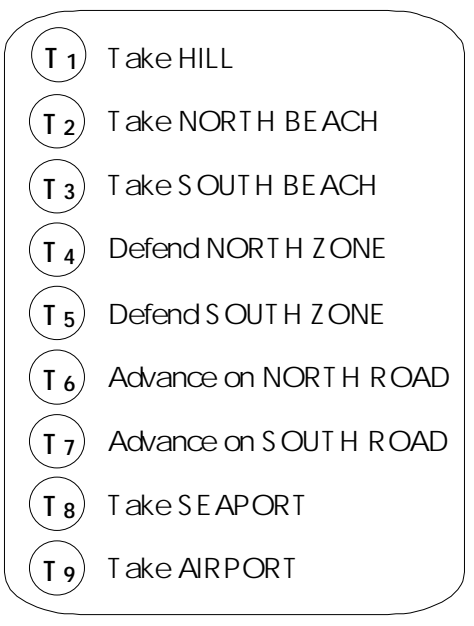

Figure 2. Geographical constraints and tasks of a simplified mission in the Distributed Dynamic Decisionmaking (DDD) simulator

\section{TABLE I}

Illustration of Mission Task Parameters

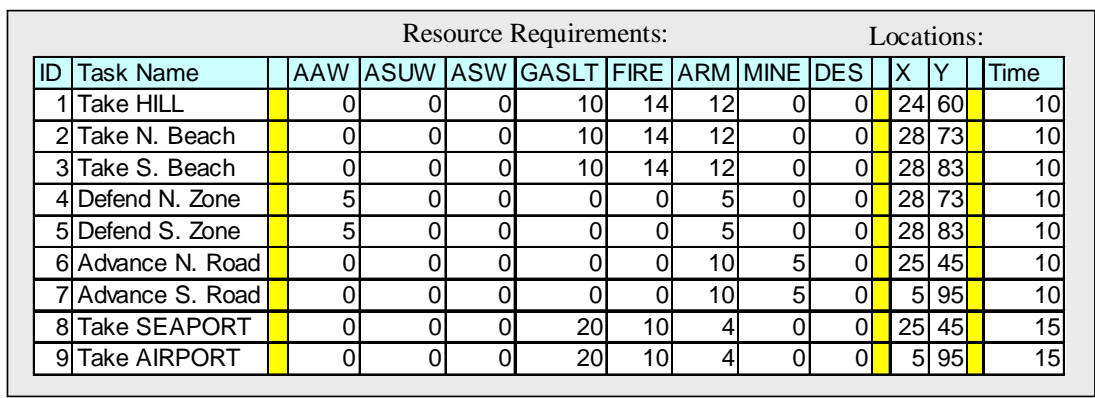




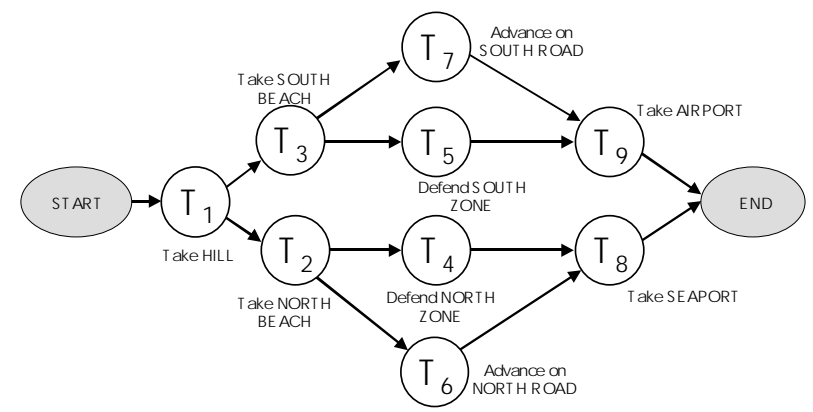

Figure 3: Mission Precedence Task Graph

TABLE II

Illustration of Platform Parameters

\begin{tabular}{|r|l|r|r|r|r|r|r|r|r|r|r|}
\hline \multicolumn{7}{|c|}{ Resource Capabilities: } \\
\hline ID & Platform Name & AAW & ASUW & ASW & GASLT & FIRE & ARM & MINE & DES & Velocity \\
\hline 1 & DDG & 10 & 10 & 1 & 0 & 9 & 5 & 0 & 0 & 2 \\
\hline 2 & FFG & 1 & 4 & 10 & 0 & 4 & 3 & 0 & 0 & 2 \\
\hline 3 & CG & 10 & 10 & 1 & 0 & 9 & 5 & 0 & 0 & 2 \\
\hline 4 & ENG & 0 & 0 & 0 & 2 & 0 & 0 & 5 & 0 & 4 \\
\hline 5 & INFA & 1 & 0 & 0 & 10 & 2 & 2 & 1 & 0 & 1.35 \\
\hline 6 & SD & 5 & 0 & 0 & 0 & 0 & 0 & 0 & 0 & 4 \\
\hline 7 & AHI & 3 & 4 & 0 & 0 & 6 & 10 & 1 & 0 & 4 \\
\hline 8 & CAS1 & 1 & 3 & 0 & 0 & 10 & 8 & 1 & 0 & 4 \\
\hline 9 & CAS2 & 1 & 3 & 0 & 0 & 10 & 8 & 1 & 0 & 4 \\
\hline 10 & CAS3 & 1 & 3 & 0 & 0 & 10 & 8 & 1 & 0 & 4 \\
\hline 11 & VF1 & 6 & 1 & 0 & 0 & 1 & 1 & 0 & 0 & 4.5 \\
\hline 12 & VF2 & 6 & 1 & 0 & 0 & 1 & 1 & 0 & 0 & 4.5 \\
\hline 13 & VF3 & 6 & 1 & 0 & 0 & 1 & 1 & 0 & 0 & 4.5 \\
\hline 14 & SMC & 0 & 0 & 0 & 0 & 0 & 0 & 10 & 0 & 2 \\
\hline 15 & TARP & 0 & 0 & 0 & 0 & 0 & 0 & 0 & 6 & 5 \\
\hline 16 & SAT & 0 & 0 & 0 & 0 & 0 & 0 & 0 & 6 & 7 \\
\hline 17 & SOF & 0 & 0 & 0 & 6 & 6 & 0 & 1 & 10 & 2.5 \\
\hline 18 & INF(AAAV-1) & 1 & 0 & 0 & 10 & 2 & 2 & 1 & 0 & 1.35 \\
\hline 19 & INF(AAAV-2) & 1 & 0 & 0 & 10 & 2 & 2 & 1 & 0 & 1.35 \\
\hline 20 & INF(MV22-1) & 1 & 0 & 0 & 10 & 2 & 2 & 1 & 0 & 1.35 \\
\hline
\end{tabular}

\subsection{Design Uncertainty}

In our 3-phase organizational design process, we construct an organization based on specific static parameters known about the organization and the environment. Unfortunately, the static assumption is potentially erroneous, since the environment is a dynamic entity evolving under many processes, sometimes not related to the organization and its activities. In addition, whereas organizational parameters may be accurately measured in advance, this might not be the case with regard to mission tasks, where the measurement errors may inhibit accurate modeling, or the task data may have been obtained as a set of random parameters. Consequently, the mission environment could "swerve" into unaccountable directions, and a fixed organization would fail. Therefore, it is necessary to design organizations to account for possible uncertainties.

The following uncertainties are the features of an organizational design problem:

a) Measurement errors: this type of uncertainty relates to errors in measuring the task data, such as task processing times, task locations, and task-resource requirements. 
b) Task Precedence errors: a task precedence graph is a plan that a commander uses to execute a mission. This plan can undergo changes during mission processing under a changing environment and/or changing organizational capabilities.

c) Task Decomposition errors: the task data for a specified mission is obtained from task decomposition graph. As stated earlier, various decomposition techniques can be used to break the mission into elementary tasks. The decomposition must be consistent with organizational goals and capabilities. Since the goals of an organization may change under changing environment and/or organizational parameters, task decomposition should be adjusted accordingly. On the other hand, in the event of uncertain information about a specific task, it may be desirable to decompose it into smaller sub-tasks to be performed separately and with better accuracy.

d) Unexpected tasks: unforeseen tasks may appear for which decision-makers have not been trained. The organization must find a way to react to such environmental changes by adjusting its strategy (and, sometimes, structure).

e) DM failures and/or platform (resource) failures: these refer to possible failures, such as physical elimination, and expendable resources.

The uncertainties listed above introduce variability in the mission and/or resources, requiring an organization to perform well in the face of mission and/or organizational variations.

\subsection{Robust Design Methodology}

Let missions $\boldsymbol{M}_{i}, i \geq 1$, be variations on the original mission $\boldsymbol{M}$ obtained randomly using the uncertainty parameters a)-d).

Definition: A mission $\boldsymbol{M}_{C}$ is called a concatenation of mission $\boldsymbol{M}_{j}$ to mission $\boldsymbol{M}_{i}$, if $\boldsymbol{M}_{C}$ consists of all tasks defined in $\boldsymbol{M}_{i}$ and $\boldsymbol{M}_{j}$, with the restriction that tasks from $\boldsymbol{M}_{i}$ must be executed before tasks $\boldsymbol{M}_{j}$ can start. That is, precedence constraints are added among all terminal tasks (or end-task - tasks with no successors) of mission $\boldsymbol{M}_{i}$ and all onset tasks (or start-tasks tasks with no predecessors) of mission $\boldsymbol{M}_{j}$. A mission $\boldsymbol{M}_{C}^{(K)}$ is called a $K$-concatenation, if it is constructed by concatenating $K$ missions $\boldsymbol{M}_{1}, \ldots, \boldsymbol{M}_{K}$ (Fig. 4).

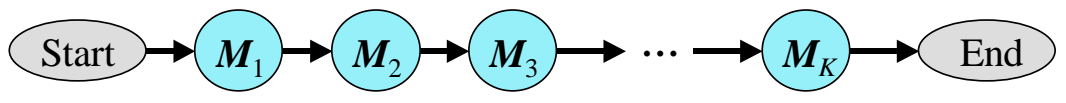

Figure 4: A schematic representation of $K$-concatenation.

An organization, designed to perform mission $\boldsymbol{M}_{C}^{(K)}$, would sequentially perform missions $\boldsymbol{M}_{i}, i=1, \ldots, K$. Therefore, such an organization's performance corresponds to an averaged performance over the set of missions $\left\{\boldsymbol{M}_{i}\right\}_{i=1}^{K}$. As missions $\boldsymbol{M}_{i}$ are random variations of the nominal mission $\boldsymbol{M}$ under environmental uncertainties, they represent the elements of a neighborhood of mission $\boldsymbol{M}$. Constructing an organization $O_{C}^{(K)}$ using our 3-phase design process to perform mission $\boldsymbol{M}_{C}^{(K)}$ would produce a robust organization (when parameter $K$ is large). Note that caution should be exercised in selecting the number of concatenations $K$, since 
the complexity of building an optimal organization for a mission $\boldsymbol{M}_{C}^{(K)}$ increases significantly with $K$.

We propose the following algorithm to find a robust organization to execute a mission $\boldsymbol{M}$ :

\section{Initialization:}

Mission $\boldsymbol{M}$; number of concatenations $K$; organization's parameters and environment's uncertainty data.

Step 1. Randomly generate missions $\boldsymbol{M}_{i}, i=1, \ldots, K$. Construct a $K$-concatenation $\boldsymbol{M}_{C}^{(K)}$.

Step 2. Apply our 3-phase design process to obtain an organization $O_{C}^{(K)}$ designed to execute mission $\boldsymbol{M}_{C}^{(K)}$.

\subsection{Example: Designing Robust Organizations}

Let us assume that the mission in the example has the following measurement errors (and these errors are the same for each task):

口 Task resource requirements errors: $\varepsilon_{R}=[2,3,2,5,4,5,2,1]$;

口 Task location errors: $\varepsilon_{L}=[5,10]$;

口 Task processing time errors: $\varepsilon_{P}=[5]$; and

a Up to 5 new tasks may appear with parameters in Table III:

TABLE III

Ranges of Parameters for Random Task

\begin{tabular}{|l|r|r|r|r|r|r|r|r|r|r|r|r|r|r|}
\hline \multicolumn{11}{|c|}{ Resource Requirements: } \\
\hline & AAW & ASUW & ASW & GASLT & FIRE & ARM & MINE & DES & X & Y & Time \\
\hline Minimum & 0 & 1 & 0 & 10 & 5 & 3 & 0 & 0 & 5 & 15 & 5 \\
\hline Maximum & 3 & 2 & 0 & 20 & 10 & 8 & 4 & 1 & 30 & 60 & 15 \\
\hline
\end{tabular}

Let us randomly generate a new mission $\boldsymbol{M}_{1}$ accounting for these uncertainties. Note that random tasks may appear anywhere inside the precedence graph. However, let us limit the number of its successors (and predecessors) to be between 1 and 3. Parameters of the mission $\boldsymbol{M}_{1}$ are shown in Table IV, and the task precedence graph in Fig.5. 
TABLE IV

Mission Task Parameters for Mission $\boldsymbol{M}_{1}$

\begin{tabular}{|c|c|c|c|c|c|c|c|c|c|c|c|c|}
\hline & & \multicolumn{6}{|c|}{ Resource Requirements: } & \multicolumn{5}{|c|}{ Locations: } \\
\hline ID & Task Name & AAW & ASUW & ASW & GASLT & FIRE & ARM & MINE & DES & $\mathrm{X}$ & $\mathrm{Y}$ & Time \\
\hline 1 & Take HILL & 0 & 0 & 1 & 15 & 17 & 11 & 0 & 0 & 19 & 55 & 10.9 \\
\hline 2 & Take N. Beach & 1 & 0 & 0 & 10 & 11 & 7 & 2 & 0 & 33 & 68 & 7.4 \\
\hline 3 & Take S. Beach & 1 & 0 & 1 & 11 & 16 & 11 & 0 & 1 & 33 & 85 & 5.5 \\
\hline 4 & Defend N. Zone & 4 & 0 & 0 & 0 & 4 & 8 & 0 & 1 & 32 & 78 & 7.6 \\
\hline 5 & Defend S. Zone & 6 & 1 & 1 & 0 & 0 & 9 & 0 & 1 & 33 & 82 & 5.9 \\
\hline 6 & Advance N. Road & 0 & 1 & 0 & 3 & 4 & 6 & 7 & $\overline{0}$ & 28 & 50 & 10.5 \\
\hline 7 & Advance S. Road & 0 & 0 & 0 & 3 & 3 & 13 & 7 & 1 & 9 & 98 & 8.2 \\
\hline 8 & Take SEAPORT & 0 & 1 & 0 & 22 & 13 & 0 & 0 & 0 & 23 & 49 & 13.2 \\
\hline 9 & Take AIRPORT & 0 & 0 & 2 & 20 & 10 & 0 & 2 & 0 & 9 & 92 & 11.2 \\
\hline 10 & Random 1 & 0 & 2 & 0 & 11 & 7 & 4 & 1 & 1 & 7 & 40 & 8.8 \\
\hline 11 & Random 2 & 1 & 1 & 0 & 20 & 9 & 8 & 2 & 0 & 17 & 47 & 5.7 \\
\hline 12 & Random 3 & 1 & 2 & 0 & 12 & 6 & 3 & 2 & 0 & 28 & 34 & 5.7 \\
\hline 13 & Random 4 & 3 & 1 & 0 & 11 & 8 & 3 & 4 & 1 & 20 & 54 & 10.4 \\
\hline
\end{tabular}

Let the organization $O$ be designated "optimal" (that is, constructed by optimizing the objective function using our 3-phase design process) to execute mission $\boldsymbol{M}$. We would like to evaluate its performance on mission $\boldsymbol{M}_{1}$. For this purpose, we also design "optimal" organization $O_{1}$ to execute $\boldsymbol{M}_{1}$. The corresponding DM-platform allocation and the task coordination is shown in Fig.6. Results show that the structure of organization $O$ could not sustain changes in the mission (mainly, changes in coordination among DMs). This is supported by the performance data comparison for organizations $O$ and $O_{1}$.

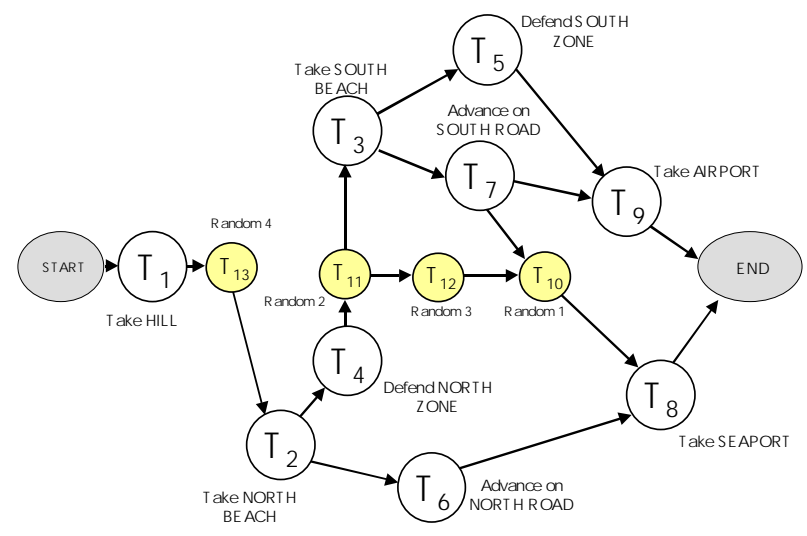

Figure 5: Mission $\boldsymbol{M}_{1}$ Precedence Task Graph

We have:

$$
\begin{aligned}
& \underline{\Pi}\left(O, M_{1}\right)=[0.2718,0.194,0.5187,1.2194,0.4335,0.7263,1.5755,1.8282] \\
& \underline{\Theta}\left(O, M_{1}\right)=[0.433,0.8326,0.8564,0.4086,0.8508,0.8933] \\
& \underline{\Pi}\left(O_{1}, M_{1}\right)=[0.2629,0.2167,0.5783,1.1284,0.4030,0.5570,0.8274,0.9547] \\
& \underline{\Theta}\left(O_{1}, M_{1}\right)=[0.359,0.3786,0.3786,0.1302,0.1302,0.1302] \\
& \left\|\underline{\Pi}\left(O, M_{1}\right)\right\|_{2}=2.8996,\left\|\underline{\Theta}\left(O, M_{1}\right)\right\|_{2}=1.8174 \\
& \left\|\underline{\Pi}\left(O_{1}, M_{1}\right)\right\|_{2}=1.9474,\left\|\underline{\Theta}\left(O_{1}, M_{1}\right)\right\|_{2}=0.6829
\end{aligned}
$$




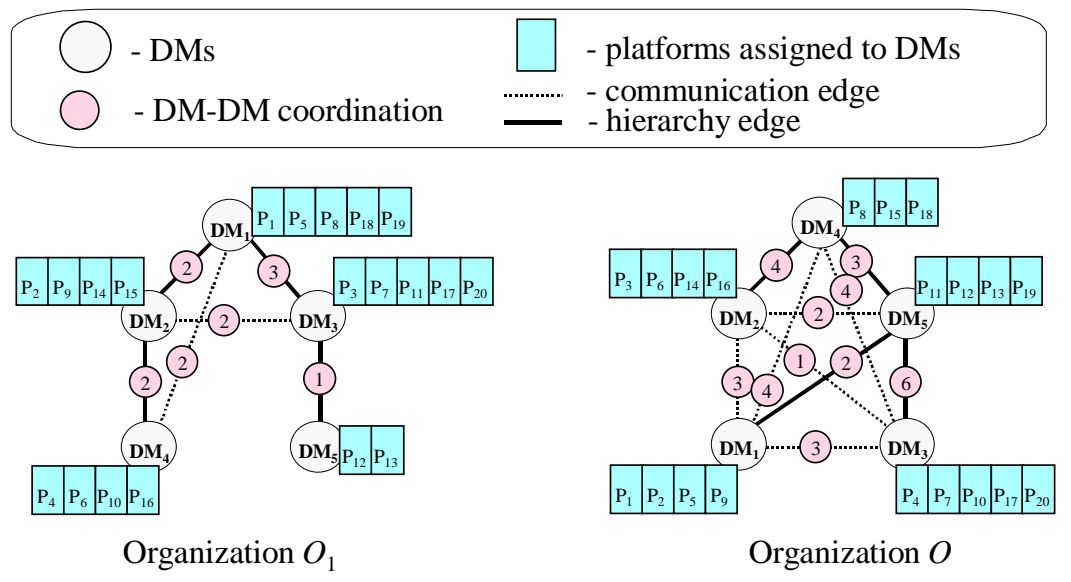

Figure 6: Organizational Structures of Organizations $O_{1}$ and $O$ on mission $\boldsymbol{M}_{1} ; W^{I}=W^{E}=1$

The performance deterioration in organization designed for mission $\boldsymbol{M}$ while executing mission $\boldsymbol{M}_{1}$ is due to the fragility of its structure. This example clearly shows the need for a robust structure. For instance, when we build an organization $O_{5}$ (Fig.7) on 5 concatenations of missions $\boldsymbol{M}_{i}, i=1,2,3,4,5$ from a neighborhood of mission $\boldsymbol{M}$, its performance on mission $\boldsymbol{M}_{1}$ is:

$$
\begin{aligned}
& \underline{\Pi}\left(O_{5}, M_{1}\right)=[0.2521,0.2618,0.6906,1.5538,0.4088,0.8225,1.4266,1.5772] \\
& \underline{\Theta}\left(O_{5}, M_{1}\right)=[0.4088,0.7178,0.7178,0.2365,0.4518,0.4518] \\
& \left\|\underline{\Pi}\left(O_{5}, M_{1}\right)\right\|_{2}=2.8965,\left\|\underline{\Theta}\left(O_{5}, M_{1}\right)\right\|_{2}=1.2891
\end{aligned}
$$

It is evident that organization $O_{5}$ is better in coordination and processing performance than organization $O$, and the degrees of congruence of these organizations are

$$
\underline{\boldsymbol{C}}\left(O, \boldsymbol{M}_{1}\right)=[0.6716,0.3758], \underline{\boldsymbol{C}}\left(O_{5}, \boldsymbol{M}_{1}\right)=[0.6723,0.5298] .
$$

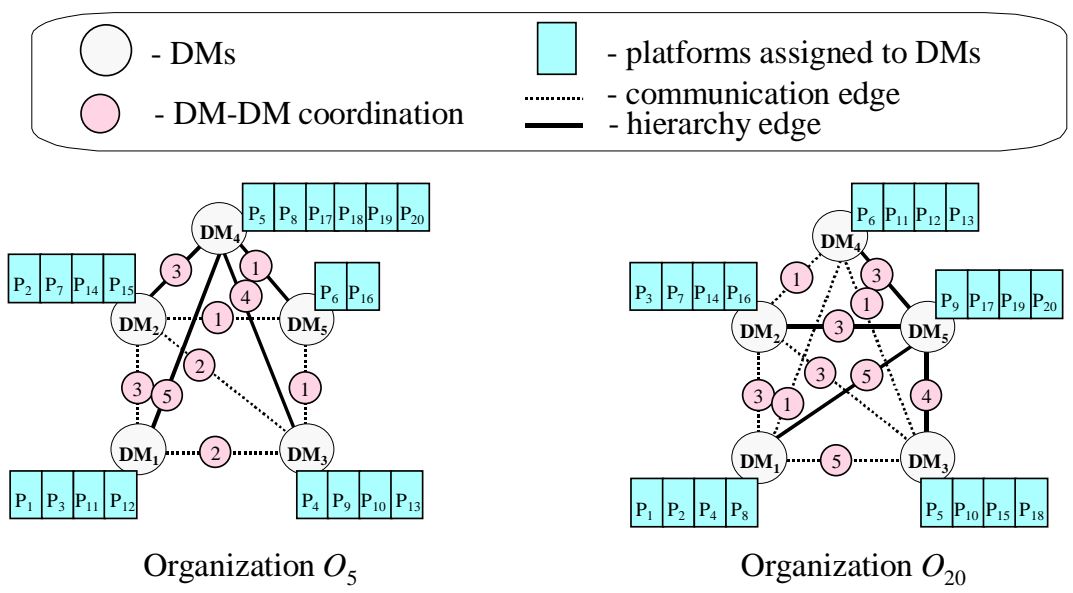

Figure 7: Organizational Structures of Robust Organizations $O_{5}$ and $O_{20}$ on mission $\boldsymbol{M}_{1} ; W^{I}=W^{E}=1$ 
Building an organization $O_{20}$ (Fig.7) on 20 concatenations of missions $\boldsymbol{M}_{i}, i=1, \ldots, 20$ in the neighborhood of the nominal mission $\boldsymbol{M}$, we would obtain even better performance in executing mission $\boldsymbol{M}_{1}$ (see Fig.8 for a comparison plot of the degrees of congruence of various organizations):

$$
\begin{aligned}
& \underline{\Pi}\left(O_{20}, M_{1}\right)=[0.2249,0.2390,0.6430,1.5300,0.4473,0.6900,1.0623,1.3407] \\
& \underline{\Theta}\left(O_{20}, M_{1}\right)=[0.3869,0.4791,0.5763,0.1566,0.2312,0.2510] \\
& \left\|\underline{\Pi}\left(O_{20}, M_{1}\right)\right\|_{2}=2.5425,\left\|\underline{\Theta}\left(O_{20}, M_{1}\right)\right\|_{2}=0.9232, \underline{\boldsymbol{C}}\left(O_{20}, \boldsymbol{M}_{1}\right)=[0.7659,0.7397]
\end{aligned}
$$

Figure 8 shows the corresponding congruence data for various organizations for missions $\boldsymbol{M}$ and $\boldsymbol{M}_{1}$. The performance data for mission $\boldsymbol{M}$ is:

$$
\begin{aligned}
& \underline{\Pi}(O, M)=[0.0577,0.2475,0.3847,0.6710,0.6076,1.0424,1.5088,1.6709] \\
& \underline{\Theta}(O, M)=[0.5133,0.6432,0.6432,0.3202,0.3202,0.3202] \\
& \underline{\Pi}\left(O_{1}, \boldsymbol{M}\right)=[0.0288,0.2475,0.3847,1.1841,0.9342,1.4789,2.0780,3.0606] \\
& \underline{\Theta}\left(O_{1}, \boldsymbol{M}\right)=[0.7878,1.2987,1.4919,0.4726,0.6477,0.6477] \\
& \underline{\Pi}\left(O_{5}, \boldsymbol{M}\right)=[0.0577,0.2668,0.3999,1.0733,0.8537,1.4516,2.3451,2.7418] \\
& \underline{\Theta}\left(O_{5}, \boldsymbol{M}\right)=[0.8128,1.3137,1.3667,0.4227,0.5764,0.5764] \\
& \underline{\Pi}\left(O_{20}, \boldsymbol{M}\right)=[0.0288,0.288,0.4183,1.2338,0.8725,1.4451,1.4694,2.4639] \\
& \underline{\Theta}\left(O_{20}, \boldsymbol{M}\right)=[0.7510,0.8201,1.0843,0.2306,0.2306,0.2306]
\end{aligned}
$$
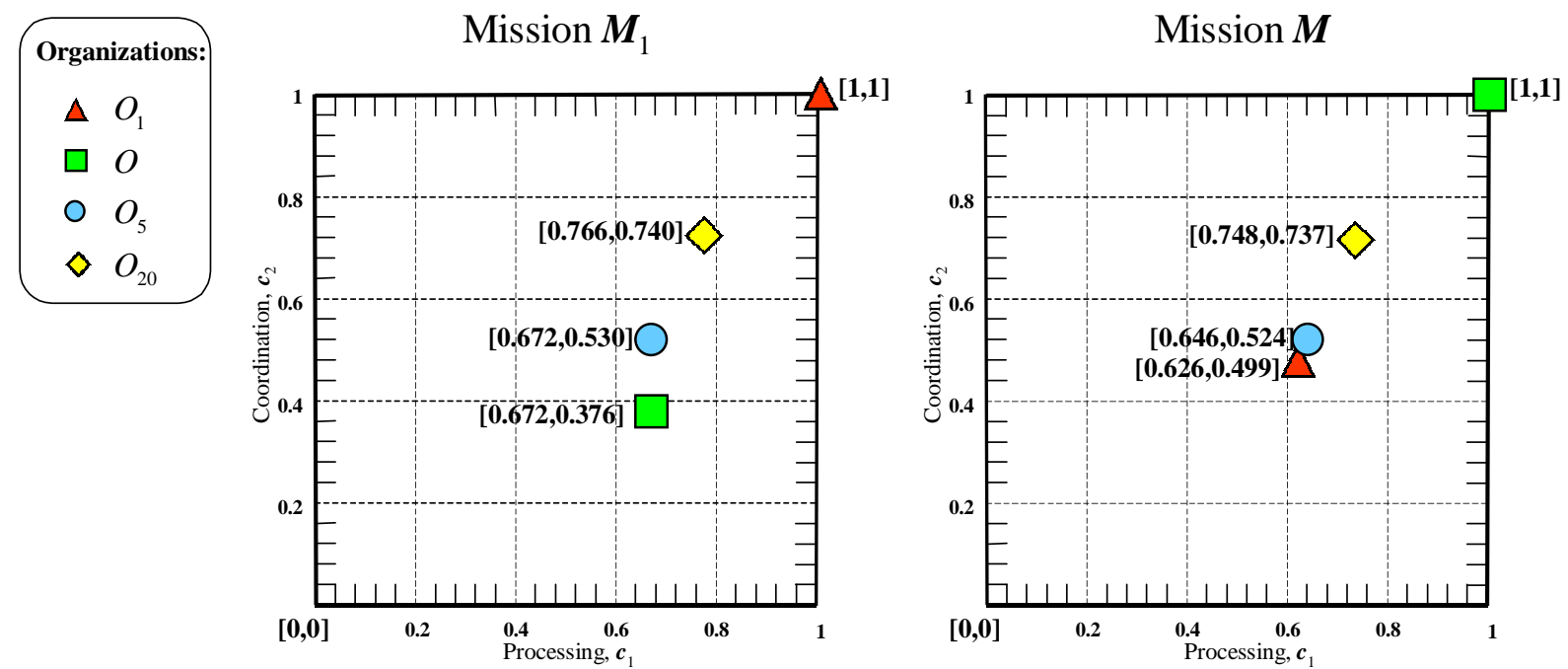

Figure 8: Degrees of Congruence of Various Organizations

Computing the corresponding norms, we obtain the measures of congruence as 
$\underline{\boldsymbol{C}}(O, \boldsymbol{M})=[1,1], \underline{\boldsymbol{C}}\left(O_{1}, \boldsymbol{M}\right)=[0.626,0.499], \underline{\boldsymbol{C}}\left(O_{5}, \boldsymbol{M}\right)=[0.646,0.524], \underline{\boldsymbol{C}}\left(O_{20}, \boldsymbol{M}\right)=[0.748,0.737]$

It is evident that organizations $O$ (optimal for mission $\boldsymbol{M}$ ) and $O_{1}$ (optimal for $\boldsymbol{M}_{1}$ ) are fragile; their performance (correspondingly, the degree of congruence) is low on each other's missions. On the other hand, organizations $O_{5}$ and $O_{20}$ (designed to be robust) maintain their high levels of performance and degrees of congruence (with organization $O_{20}$ having the better performance, since it is the outcome of a larger number of concatenations used in its construction). These results clearly show the need for robust design and indicate the fragility of organizations matched to their missions.

We display our multi-parameter performance measures in the form of a radar (or spider) plot (a processing and coordination performance for organizations $O, O_{1}$ and $O_{5}$ on mission $\boldsymbol{M}_{1}$ is shown in Fig.9). The idea of a radar plot is similar to that of the star plot (see [Fienberg, 1979], [Keller and Keller, 1993]). In a radar plot, radii stretching out from the center of a circle represent the data. Each radius denotes an element of a multidimensional vector corresponding to the performance of an organization. The value of a performance parameter for each organization is displayed by points of different shapes, colors, or both. However, when there are too many vectors, the data pattern will be concealed.

We consider two displays - coordination performance plot (including coordination measures) and processing performance plot (including activity and information exchange measures). It can be seen from Fig.9 that the performance of organization $O_{1}$ is the best, while organization $O_{5}$ provides a trade-off between a fragile organization $O$ and optimally matched organization $O_{1}$. The degradation in performance of organization $O$ stems from poor coordination, where unforeseen changes in the mission alter the pattern of inter-DM interactions. Evidently, organization $O$ does not have the necessary redundancies to cope with this change.
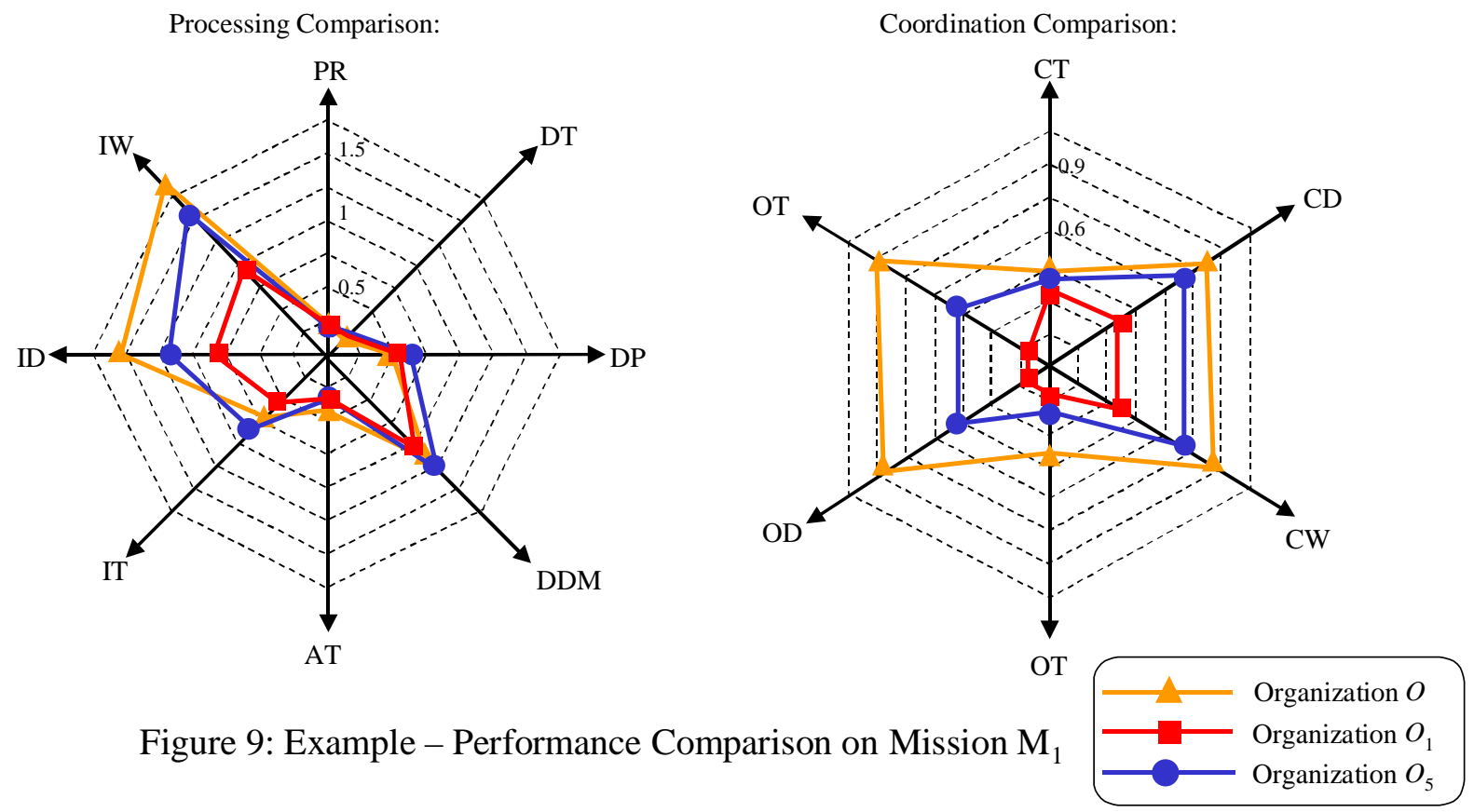

Figure 9: Example - Performance Comparison on Mission $\mathrm{M}_{1}$

\section{Structural Adaptation}




\subsection{Adaptation Cost}

In the following, we consider only organizations with the same resource capabilities and same number of available DMs. Clearly, this can be extended to the general case. We define the cost of adaptation between two organizations as the difference between their structures. The structure of an organization is defined via two matrices:

(i) DM-platform assignment matrix $\mathrm{A}=\left[y_{k m}\right], k=1, \ldots, D ; m=1, \ldots, R$; and

(ii) DM-DM hierarchy tree matrix $\mathrm{E}=\left[e_{i j}\right], i, j=1, \ldots, D$.

These matrices define organizations with respect to permutations of DM ordering. That is, if DMs are indistinguishable, then exchanging the assignments of such DMs as well as positions in the hierarchy would produce the same organization. Clearly, matrices $\mathrm{A}$ and $\mathrm{E}$ would change. Accordingly, we define two phases of structural adaptation, and their costs:

1. DM-platform reassignment - defined by matrix $\mathrm{A}$; the cost to remove or assign a platform to a DM is $\boldsymbol{W}_{\mathrm{P}}$; and

2. Hierarchy reassignment - defined by matrix $\mathrm{E}$; the cost of removing or adding a link in the hierarchy is $\boldsymbol{W}_{\mathrm{H}}$.

Following the above logic, the total cost of adaptation is equal to the sum of DM-platform reassignment cost and Hierarchy reassignment cost. The following definition formalizes the notion of adaptation cost:

Definition: The cost of adaptation from organization $O_{1}$ to organization $\mathrm{O}_{2}$ having the same resource capabilities and same number of available DMs, represented by their corresponding matrices $\mathrm{A}^{1}=\left[y_{k m}^{1}\right], \mathrm{E}^{1}=\left[e_{i j}^{1}\right]$ and $\mathrm{A}^{2}=\left[y_{k m}^{2}\right], \mathrm{E}^{2}=\left[e_{i j}^{2}\right]$, is defined as

$$
C_{12}=\min _{\Phi}\left(\boldsymbol{W}_{\mathrm{P}} \cdot\left\|\mathrm{A}^{1}-\Phi \cdot \mathrm{A}^{2}\right\|_{1}+\boldsymbol{W}_{\mathrm{H}} \cdot\left\|\mathrm{A}^{1}-\Phi \cdot \mathrm{A}^{2}\right\|_{1}\right)
$$

where $\Phi$ is a permutation matrix (for example, a matrix $\Phi \cdot \mathrm{A}^{2}$ is obtained from $\mathrm{A}^{2}$ via row permutations), and $\|A\|_{1}=\left\|\left[a_{i j}\right]\right\|_{1}=\sum_{i, j}\left|a_{i j}\right|$.

Let the permutation matrix $\Phi$ correspond to the permutation $\left[\begin{array}{ccccc}1 & 2 & 3 & \ldots & D \\ j_{1} & j_{2} & j_{3} & \ldots & j_{D}\end{array}\right]$. We have: $\sum_{k=1}^{D} \sum_{m=1}^{R}\left|y_{k m}^{1}-y_{j_{k} m}^{2}\right|$ is the number of platform assignments and removals for corresponding matrices, and $\sum_{k=1}^{D} \sum_{i=1}^{D}\left|e_{k i}^{1}-e_{j_{k} i}^{2}\right|$ is the number of hierarchy link removals and additions. Hence: 


$$
\begin{aligned}
& \boldsymbol{W}_{\mathrm{P}} \cdot\left\|\mathrm{A}^{1}-\Phi \cdot \mathrm{A}^{2}\right\|_{1}+\boldsymbol{W}_{\mathrm{H}} \cdot\left\|\mathrm{A}^{1}-\Phi \cdot \mathrm{A}^{2}\right\|_{1}=\boldsymbol{W}_{\mathrm{P}} \cdot \sum_{k=1}^{D} \sum_{m=1}^{R}\left|y_{k m}^{1}-y_{j_{k} m}^{2}\right|+\boldsymbol{W}_{\mathrm{H}} \cdot \sum_{k=1}^{D} \sum_{i=1}^{D}\left|e_{k i}^{1}-e_{j_{k} i}^{2}\right| \\
& =\sum_{k=1}^{D}\left(\boldsymbol{W}_{\mathrm{P}} \cdot \sum_{m=1}^{R}\left|y_{k m}^{1}-y_{j_{k} m}^{2}\right|+\boldsymbol{W}_{\mathrm{H}} \cdot \sum_{i=1}^{D}\left|e_{k i}^{1}-e_{j_{k} i}^{2}\right|\right) \\
& =\sum_{k=1}^{D} \sum_{r=1}^{R} \mu_{k r}\left(\boldsymbol{W}_{\mathrm{P}} \cdot \sum_{m=1}^{R}\left|y_{k m}^{1}-y_{r m}^{2}\right|+\boldsymbol{W}_{\mathrm{H}} \cdot \sum_{i=1}^{D}\left|e_{k i}^{1}-e_{r i}^{2}\right|\right)
\end{aligned}
$$

where $\mu_{k r}$ are assignment variables, such that $\mu_{k r}=\left\{\begin{array}{l}1, \text { if } r=j_{k} \\ 0, \text { otherwise }\end{array}\right.$.

Therefore, the problem of finding the cost to adapt from organization $O_{1}$ to organization $O_{2}$ becomes:

$$
\begin{aligned}
& \min _{\mu_{k r}} \sum_{k=1}^{D} \sum_{r=1}^{R} \mu_{k r}\left(\boldsymbol{W}_{\mathrm{P}} \cdot \sum_{m=1}^{R}\left|y_{k m}^{1}-y_{r m}^{2}\right|+\boldsymbol{W}_{\mathrm{H}} \cdot \sum_{i=1}^{D}\left|e_{k i}^{1}-e_{r i}^{2}\right|\right) \\
& \text { such that } \sum_{k=1}^{D} \mu_{k r}=\sum_{r=1}^{D} \mu_{k r}=1
\end{aligned}
$$

This is equivalent to the assignment problem with the cost of assigning an object $k$ to a bin $r$ equal to

$$
\boldsymbol{W}_{\mathrm{P}} \cdot \sum_{m=1}^{R}\left|y_{k m}^{1}-y_{r m}^{2}\right|+\boldsymbol{W}_{\mathrm{H}} \cdot \sum_{i=1}^{D}\left|e_{k i}^{1}-e_{r i}^{2}\right| .
$$

This problem can be solved by applying the auction algorithm [Bertsekas, 1998].

\subsection{Adaptation Process}

Assume that an organization is to be designed to process a mission $\boldsymbol{N}$, a concatenation of missions $\boldsymbol{M}_{1}, \ldots, \boldsymbol{M}_{K}$. Each of the missions $\boldsymbol{M}_{i}$ may represent elementary environment transformations (for example, events that occur and change the environment) and their concatenated sequence is viewed as a morphing process of mission $\boldsymbol{N}$ through missions $\boldsymbol{M}_{1}, \ldots, \boldsymbol{M}_{K}$. For each mission $\boldsymbol{M}_{i}$ and organization $O_{j}$, we can calculate the performance

parameter $p_{j i}=\left\|[1,1]-\underline{\boldsymbol{C}}\left(O_{j}, \boldsymbol{M}_{i}\right)\right\|_{2}$. This parameter can be viewed as congruence mismatch (the smaller $p_{j i}$, the more congruent the corresponding organization $O_{j}$ is to mission $\boldsymbol{M}_{i}$ ). We say that organization $O_{j}$ is congruent to this mission if parameter $p_{j i}$ is below a specified congruence mismatch threshold (see Fig.10). For each mission $\boldsymbol{M}_{i}$, we consider only its congruent organizations. We construct a Viterbi trellis (Fig.11) with each stage $i$ corresponding to a mission $\boldsymbol{M}_{i}$, with paths leading only to (from) nodes representing organizations congruent with this mission. The value of the path between two organizations $O_{j}$ and $O_{l}$ (both congruent for missions $\boldsymbol{M}_{i}$ and $\boldsymbol{M}_{i+1}$ ) is equal to $C_{j l}$ - the cost of structural adaptation from $O_{j}$ to $O_{l}$ (obtained by solving an assignment problem). To obtain the least-cost adaptation strategy (Fig.12), we find the least-cost terminal path via Viterbi algorithm, a well-known technique in digital communications [Forney, 1973]. The Viterbi algorithm is a recursive optimal solution to 
the problem of finding the shortest path in a trellis (for more details, see [Forney, 1973], [Bertsekas, 1995], [Busacker and Saaty, 1965]).

The following algorithm formalizes our approach for designing adaptive organizations:

Step 1. Find the congruence parameter $p_{j i}$ for each organization $O_{j}$ and mission $\boldsymbol{M}_{i}$.

Step 2. Construct a trellis using only congruent organizations as described above.

Step 3. Find the least-cost ("shortest") path via Viterbi algorithm, and specify adaptation triggers.

\subsection{Example: Organizational Adaptation}

Let mission $N$ be a 5-concatenation of missions in a neighborhood of the original mission $\boldsymbol{M}$, that is, $\quad \boldsymbol{N}=\boldsymbol{M}_{1} \rightarrow \boldsymbol{M}_{2} \rightarrow \boldsymbol{M}_{3} \rightarrow \boldsymbol{M}_{4} \rightarrow \boldsymbol{M}_{5}$. We illustrate the adaptation process on an example where the organization can change among the following 4 organizational structures before processing each mission $\boldsymbol{M}_{i}$ :

1. Organization $O$ - organization designed on 5 concatenations of the original mission (different from $\boldsymbol{N}$ ) - in a sense, "robust" organization.

2. Organization $O_{1}$ - same as described in section 6.4.

3. Organization $O^{N}$ - optimal for mission $N$ (note: this organization should be generally better than $O$ since its 5 concatenations are precisely the mission that is being executed).

4. Organization $O_{i}^{M}$ - optimal for mission $\boldsymbol{M}_{i}$.

The cost of changing the structure among these organizations is shown in Table V. Figure 10 shows the star-plot of organization's congruence mismatch with each radii corresponding to a different mission. The idea of displaying the data in this fashion is related to object displays. Promoted by Wickens [Wickens, 1986] as a method of grouping similar information into an easily interpretable system state display, object displays have received considerable attention with regard to their usefulness compared to more traditional data rendering techniques (e.g., [Coury and Purcell, 1988], [Blick et al., 2000]). In our case, the web-plot allows us to display and compare the congruence mismatch parameter of different organizations executing the same mission. The data for Fig.10 is shown in Table V.

\section{TABLE V}

Example: Cost of Adaptation and Congruence Mismatch (for Cost Weights $W_{\mathrm{P}}=W_{\mathrm{H}}=1$ )

\begin{tabular}{|c|c|c|c|c|c|c|c|c|}
\hline \multicolumn{9}{|c|}{ Cost of Adaptation $C_{i j}$ : } \\
\hline & $O$ & $O_{1}$ & $0^{N}$ & $\rho_{1}^{M}$ & $O_{2}^{M}$ & $O_{3}^{M}$ & $O_{4}^{M}$ & $O_{5}^{M}$ \\
\hline$O$ & 0 & 22 & 20 & 24 & 24 & 22 & 22 & 24 \\
\hline$O_{1}$ & 22 & 0 & 20 & 22 & 28 & 24 & 26 & 28 \\
\hline$O^{N}$ & 20 & 20 & 0 & 20 & 20 & 22 & 26 & 26 \\
\hline$O_{1}^{M}$ & 24 & 22 & 20 & & 30 & & & \\
\hline$O_{2}^{M}$ & 24 & 28 & 20 & & & 28 & & \\
\hline$O_{3}^{M}$ & 22 & 24 & 22 & & & & 28 & \\
\hline$O_{4}^{M}$ & 22 & 26 & 26 & & & & & 26 \\
\hline$O_{5}^{M}$ & 24 & 28 & 26 & & & & & \\
\hline
\end{tabular}

\begin{tabular}{|c|c|c|c|c|c|}
\hline & $M_{1}$ & $M_{2}$ & $M_{3}$ & $M_{4}$ & $M_{5}$ \\
\hline$O$ & 0.6586 & 0.5019 & 0.6537 & 0.6346 & 0.7127 \\
\hline$O_{1}$ & 0.5863 & 0.7444 & 0.6105 & 0.7777 & 0.4741 \\
\hline$O^{N}$ & 0.4356 & 0.5929 & 0.6319 & 0.731 & 0.6362 \\
\hline
\end{tabular}


As we can see from the adaptation strategy, our organization could not sustain its performance and had to adapt to complete sub-mission $\boldsymbol{M}_{5}$. Therefore, the events responsible for the corresponding environmental changes are identified as adaptation triggers (changes that require adaptation). Specifically, it was found that in this example the change between missions $\boldsymbol{M}_{4}$ and $\boldsymbol{M}_{5}$ triggering the adaptation was the precedence constraints variation: the task precedence graph became "flat" in $\boldsymbol{M}_{5}$.

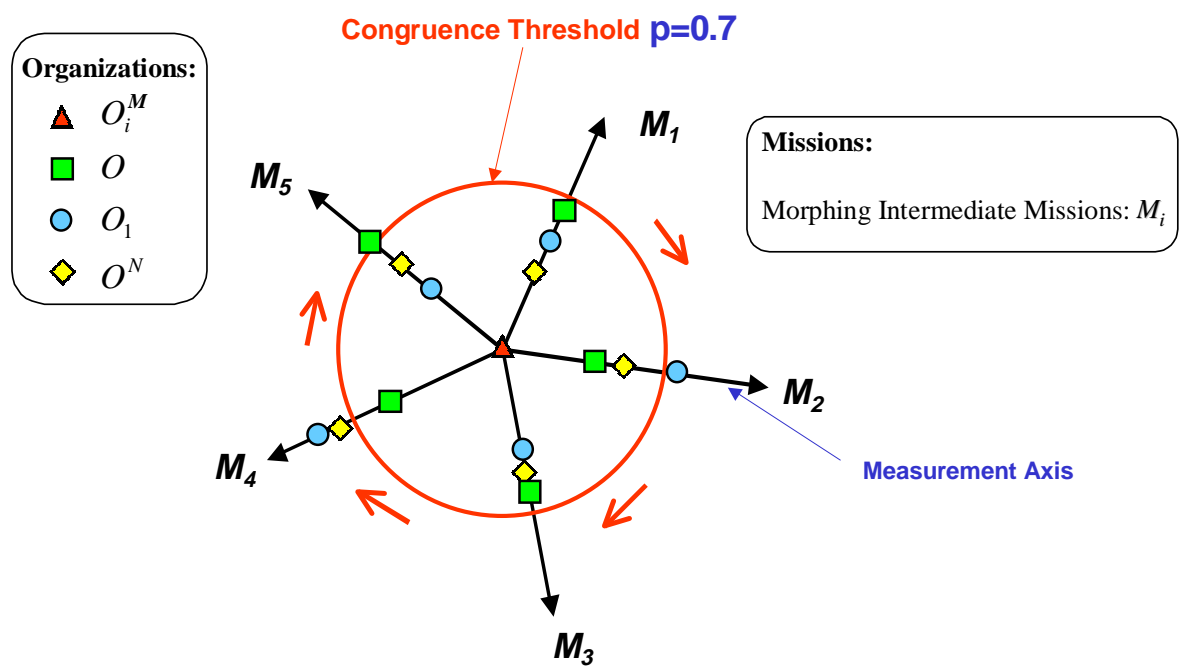

Figure 10: Adaptation Process - Congruence Threshold

As stated earlier, the Viterbi algorithm is a recursive optimal solution to the problem of finding the shortest path in an acyclic graph with trellis structure. This algorithm is essentially a forward search method that allows us to save both memory and computation in computing the shortest path. Figure 11 shows the shortest-path found by the Viterbi algorithm. At each stage of the trellis, the shortest path is found to each of its nodes from the nodes at the previous stage, the corresponding edge is marked, and the length of the path is updated. At the last stage, the terminal path with the shortest length is selected (Fig.12).

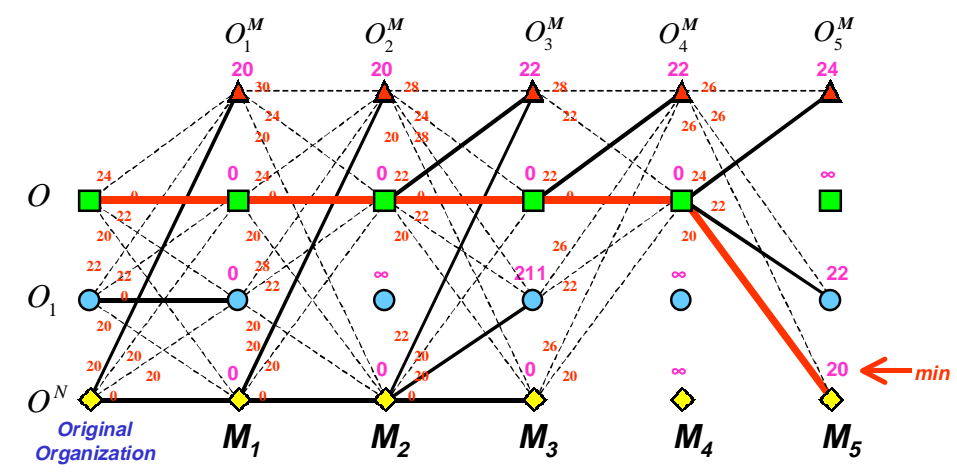

Figure 11: Adaptation Process - Strategy Selection 


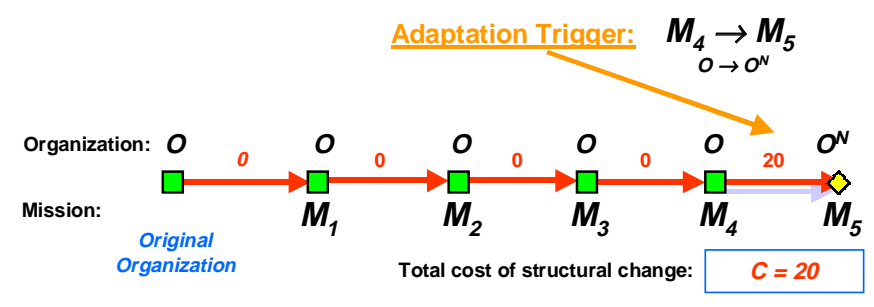

Figure 12: Example of Adaptation Strategy

\section{Summary and Future Extensions}

In this paper, we analyzed several strategies for cost-effective design of organizations to efficiently process missions having various degrees of uncertainty. Specifically, we illustrated the design of robust and adaptive organizations and discussed the corresponding performance and design trade-offs. We defined a set of performance measures to generate the objective function(s) for our design process and to analyze the sensitivity of organizational performance to changes in the mission and/or organization parameters. The notion of structural congruence between a mission and an organization has been generalized to define a measure of dynamic congruence based on multiple performance measures; it can serve as a criterion to signal the need for structural reconfiguration.

There are several extensions to this research. For example, the performance-based congruence measure introduced in this paper needs to be extended to include the structural and process (i.e., scheduling and resource allocation) match between two organizations as well. The structural adaptation cost can serve as a measure of structural incongruence between two organizations because the smaller the cost greater is the congruence. This notion currently includes DMplatform reassignment cost and hierarchy reassignment cost. It is straightforward to include temporal congruence associated with task scheduling as well.

Another extension is the inclusion of DM-related adaptation triggers. One potential adaptation trigger (and thus a cause for a structural misfit between an organization and a mission) is the mismatch between DM's dynamic capacity for task processing and the operational requirements placed on the DM. A potential degradation in DM's capacity can be predicted by monitoring the DM's psycho-physiological conditions (e.g. alertness, confusion, stress, load, fatigue, etc., which are functions of operational tempo and individual task schedules). The imbalance in the load placed on different team members may result in excessive burden on some of the DMs, increasing their stress levels and degrading their performance. Thus, it may lead to task processing inefficiencies and errors that can prove disastrous. Predicting these conditions before their onset would allow prevention of those effects, which is a key in effective team management and adaptation.

The analytic methods, applications, and measures illustrated in the paper form the basis for current research on organizational design and adaptation. 


\section{Appendix}

The performance measures used in section 2.4.1 are defined for organization's elements - DMs and/or platforms. After the dynamic measures for each element are evaluated (over the entire mission), we then compute their time-averages. If a dynamic measure is represented by a function $\varphi(t), t \in[0, Y]$, then its time-average is equal to $\frac{1}{Y} \int_{0}^{Y} \varphi(\theta) d \theta$. Because each measure corresponds to a staircase function, we obtain: $\frac{1}{Y} \int_{0}^{Y} \varphi(\theta) d \theta=\frac{1}{Y} \sum_{k=0}^{Q-1} \varphi\left(\theta_{k}\right)\left(\theta_{k+1}-\theta_{k}\right)$, where $0=\theta_{0}<\theta_{1}<\theta_{2}<\ldots<\theta_{Q-1}<\theta_{Q}=Y$ represent points of discontinuity for $\varphi(t), t \in[0, Y]$.

To analyze the distribution of the measured parameters in an organization and to compare organizational elements, one needs to consider both the mean and the variance (sample mean and sample variance) of the corresponding measures. The same pertains to the measures defined for tasks. Given an array $\underline{\boldsymbol{a}}=\left[\boldsymbol{a}_{1}, \ldots, \boldsymbol{a}_{N}\right]^{T}$ of values for $N$ elements, we can calculate a measure called efficiency, defined as follows:

$\mu(\underline{\boldsymbol{a}})+\sigma(\underline{\boldsymbol{a}})=\operatorname{mean}(\underline{\boldsymbol{a}})+\operatorname{std} v(\underline{\boldsymbol{a}})$, where

$$
\mu(\underline{\boldsymbol{a}})=\frac{1}{N} \sum_{k=1}^{N} \boldsymbol{a}_{k}, \sigma(\underline{\boldsymbol{a}})=\left(\frac{1}{N-1} \sum_{n=1}^{N}\left[\boldsymbol{a}_{n}-\mu(\underline{\boldsymbol{a}})\right]^{2}\right)^{1 / 2}=\left(\frac{1}{N-1} \sum_{n=1}^{N}\left[\boldsymbol{a}_{n}-\frac{1}{N} \sum_{k=1}^{N} \boldsymbol{a}_{k}\right]^{2}\right)^{1 / 2} .
$$

\section{A. Activity}

- Platform routing efficiency per DM:

$$
P R=\mu\left(\left[\frac{1}{R} \sum_{k=1}^{N} y_{k m} \frac{p t_{k}}{p p_{k}}\right]_{m=1}^{D}\right)+\sigma\left(\left[\frac{1}{R} \sum_{k=1}^{N} y_{k m} \frac{p t_{k}}{p p_{k}}\right]_{m=1}^{D}\right)
$$

- Task delay efficiency:

$$
D T=\mu\left(\left[\frac{1}{Y} d t_{i}\right]_{i=1}^{N}\right)+\sigma\left(\left[\frac{1}{Y} d t_{i}\right]_{i=1}^{N}\right)
$$

- Platform delay efficiency:

$$
D P=\mu\left(\left[\frac{1}{Y} d p_{m}\right]_{m=1}^{R}\right)+\sigma\left(\left[\frac{1}{Y} d p_{m}\right]_{m=1}^{R}\right)
$$

- DM delay efficiency:

$$
D D=\mu\left(\left[\frac{1}{Y} d d_{k}\right]_{k=1}^{D}\right)+\sigma\left(\left[\frac{1}{Y} d d_{k}\right]_{k=1}^{D}\right)
$$

- DM-task activity efficiency:

$$
A T=\mu\left(\left[\frac{1}{Y} \int_{0}^{Y} a t_{k}(\theta) d \theta\right]_{k=1}^{D}\right)+\sigma\left(\left[\frac{1}{Y} \int_{0}^{Y} a t_{k}(\theta) d \theta\right]_{k=1}^{D}\right)
$$




\section{B. Coordination}

- DM-task coordination efficiency:

$$
C T=\mu\left(\left[\frac{1}{Y} \int_{0}^{Y} c t_{k}(\theta) d \theta\right]_{k=1}^{D}\right)+\sigma\left(\left[\frac{1}{Y} \int_{0}^{Y} c t_{k}(\theta) d \theta\right]_{k=1}^{D}\right)
$$

- DM-DM coordination efficiency:

$$
C D=\mu\left(\left[\frac{1}{Y} \int_{0}^{Y} c d_{k}(\theta) d \theta\right]_{k=1}^{D}\right)+\sigma\left(\left[\frac{1}{Y} \int_{0}^{Y} c d_{k}(\theta) d \theta\right]_{k=1}^{D}\right)
$$

- DM coordination workload efficiency:

$$
C W=\mu\left(\left[\frac{1}{Y} \int_{0}^{Y} c w_{k}(\theta) d \theta\right]_{k=1}^{D}\right)+\sigma\left(\left[\frac{1}{Y} \int_{0}^{Y} c w_{k}(\theta) d \theta\right]_{k=1}^{D}\right)
$$

- DM-task overhead efficiency:

$$
O T=\mu\left(\left[\frac{1}{Y} \int_{0}^{Y} o t_{k}(\theta) d \theta\right]_{k=1}^{D}\right)+\sigma\left(\left[\frac{1}{Y} \int_{0}^{Y} o t_{k}(\theta) d \theta\right]_{k=1}^{D}\right)
$$

- DM-DM overhead efficiency:

$$
O D=\mu\left(\left[\frac{1}{Y} \int_{0}^{Y} \operatorname{od}_{k}(\theta) d \theta\right]_{k=1}^{D}\right)+\sigma\left(\left[\frac{1}{Y} \int_{0}^{Y} \operatorname{od}_{k}(\theta) d \theta\right]_{k=1}^{D}\right)
$$

- DM workload overhead efficiency:

$$
O W=\mu\left(\left[\frac{1}{Y} \int_{0}^{Y} o w_{k}(\theta) d \theta\right]_{k=1}^{D}\right)+\sigma\left(\left[\frac{1}{Y} \int_{0}^{Y} o w_{k}(\theta) d \theta\right]_{k=1}^{D}\right)
$$

\section{Information Exchange}

- DM-task information exchange efficiency:

$$
I T=\mu\left(\left[\frac{1}{Y} \int_{0}^{Y}\left[e t_{k}^{\text {out }}(\theta)+e t_{k}^{i n}(\theta)\right] d \theta\right]_{k=1}^{D}\right)+\sigma\left(\left[\frac{1}{Y} \int_{0}^{Y}\left[e t_{k}^{\text {out }}(\theta)+e t_{k}^{i n}(\theta)\right] d \theta\right]_{k=1}^{D}\right)
$$

- DM-DM information exchange efficiency:

$$
I D=\mu\left(\left[\frac{1}{Y} \int_{0}^{Y}\left[e d_{k}^{\text {out }}(\theta)+e d_{k}^{\text {in }}(\theta)\right] d \theta\right]_{k=1}^{D}\right)+\sigma\left(\left[\frac{1}{Y} \int_{0}^{Y}\left[e d_{k}^{\text {out }}(\theta)+e d_{k}^{\text {in }}(\theta)\right] d \theta\right]_{k=1}^{D}\right)
$$

- DM information exchange workload efficiency:

$$
I W=\mu\left(\left[\frac{1}{Y} \int_{0}^{Y}\left[e w_{k}^{\text {out }}(\theta)+e w_{k}^{\text {in }}(\theta)\right] d \theta\right]_{k=1}^{D}\right)+\sigma\left(\left[\frac{1}{Y} \int_{0}^{Y}\left[e w_{k}^{\text {out }}(\theta)+e w_{k}^{\text {in }}(\theta)\right] d \theta\right]_{k=1}^{D}\right)
$$




\section{References}

[Argote and Epple, 1990] Argote L. and D. Epple, "Learning curves in manufacturing", Science, Vol. 247, 1990.

[Bertsekas, 1995] Bertsekas, D.P., “Dynamic Programming and Optimal Control”, Vol. 1, 2, Athena Scientific, Belmont, MA, 1995.

[Bertsekas, 1998] Bertsekas, D.P. Network Optimization: Continuous and Discrete Models. Athena Scientific, 1998.

[Blick et al., 2000] Blick, G., J. Jensen, K. Whalen, and S. Surgenor, "The emergent features of a medical object display improve anesthesiologists' performance of simulated diagnostic tasks", Proceedings of the International Ergonomics Association 2000/Human Factors and Ergonomics Society 2000 Congress, Santa Monica, CA: Human Factors and Ergonomics Society, 4.2584.261, 2000.

[Booth, 1996] Booth, R., “Agile Manufacturing”, Engineering Management, April 1996, 105115, 1996.

[Bucheim, 2000] Bucheim, R. K., "Developing performance metrics for a design engineering department", IEEE Transactions on Engineering Management, Vol. 47, No. 3, 309-320, 2000.

[Burton and Obel, 1998] Burton, R.M. and B. Obel, Strategic Organizational Diagnosis and Design: Developing Theory for Application (2nd Ed.). Boston, MA: Kluwer Academic Publishers, 1998.

[Busacker and Saaty, 1965] Busacker, R. and T. Saaty. Finite Graphs and Networks: An Introduction with Applications. New York: McGraw-Hill, 1965.

[Cameron, 1986] Cameron, K.S., "Effectiveness as paradox: consensus and conflict in conceptions of organizational effectiveness", Management Science, Vol. 32, No. 5, 539-553, 1986.

[Carley, 1998] Carley, K.M., "Adaptive organizations and emergent forms", Proceedings of the International Conference on Multi Agent Systems, 2 -3, 1998.

[Chunming, 1995] Chunming, D., "Developing robust system designs under uncertainty: a systematic approach to design evaluation", Proceedings of Third International Symposium on Uncertainty Modeling and Analysis and Annual Conference of the North American Fuzzy Information Processing Society, 210 -215, 1995.

[Coury and Purcell, 1988] Coury, B. and J. Purcell, "The bar graph as a configurable and separable display", Proceedings of the Human Factors Society $32^{\text {nd }}$ Annual Meeting, Santa Monica, CA: Human Factors Society, 1361-1365, 1988.

[Dill and Pearson, 1991] Dill, D. and A. W. Pearson, "The self-designing organization: structure, learning, and the management of technical professionals", Technology Management : the New International Language, 33 -36, 1991. 
[Entin, 1999] Entin, E.E., "Optimized command and control architectures for improved process and performance", Proceedings of the 1999 Command \& Control Research \& Technology Symposium, NWC, Newport, RI, 1999.

[Fienberg, 1979] Fienberg, S.E., "Graphical methods in statistics", American Statisticians, 33, $165-178,1979$.

[Foisel et al., 1998] Foisel, R., V. Chevrier, and J. Haton, "Modeling adaptive organizations", Proceedings of the International Conference on Multi Agent Systems, 427 -428, 1998.

[Forney, 1973] Forney Jr., D., "The Viterbi algorithm", Proceedings of the IEEE Conference, Vol. 61, No. 3, 268-278, 1973.

[Handley et al., 1999] Handley, H.A., Z.R. Zaidi, and A.H. Levis, "The use of simulation models in model driven experimentation", Proceedings of the 1999 Command \& Control Research \& Technology Symposium, NWC, Newport, RI, 1999.

[Heller, 2000] Heller, T., "If only we'd known sooner: developing knowledge of organizational changes earlier in the product development process", IEEE Transactions on Engineering Management, Vol. 47, No. 3, 335-344, 2000.

[Hocevar et al., 1999] Hocevar, S.P., W.G. Kemple, D. Kleinman, and G. Porter, "Assessments of simulated performance of alternative architectures for command and control: the role of coordination", Proceedings of the 1999 Command \& Control Research \& Technology Symposium, NWC, Newport, RI, 1999.

[Horling et al., 1999] Horling B, et al., "Diagnosis as an integral part of multi-agent adaptability”, UMass Computer Science Technical Report 99-03, 1999.

[Kalyanaram, 1997] Kalyanaram, G., "A process design model and an application. Innovation in technology management - the key to global leadership", PICMET '97: Portland International Conference on Management and Technology, 956, 1997.

[Keller and Keller, 1993] Keller, P.R. and M.M. Keller. Visual Cues: Practical data visualization. New Jersey: IEEE Press, 1993.

[Keeney and Raiffa, 1993] Keeney, R. and H. Raiffa. Decisions with Multiple Objectives. New York, NY: Cambridge University Press, 1993.

[Kulatilaka, 1988] Kulatilaka, N., "Valuing the flexibility of flexible manufacturing systems", IEEE Transactions on Engineering Management, Vol. 35, No. 4, 250 -257, 1988.

[Lawrence and Lorsch, 1967] Lawrence P.R. and J.W. Lorsch. Organization and Environment: Managing Differentiation and Integration. Boston, MA, USA: Graduate school of business administration, 1967.

[G. Levchuk et al., 2000a] Levchuk, G.M., Y. N. Levchuk, Jie Luo, Krishna R. Pattipati, and David L. Kleinman, "Normative design of organizations - part I: mission planning", To be published, 2000. 
[G. Levchuk et al., 2000b] Levchuk, G.M., Y. N. Levchuk, Jie Luo, Krishna R. Pattipati, and David L. Kleinman, "Normative design of organizations - part II: organizational structure", To be published, 2000.

[Y. Levchuk et al., 1997] Levchuk, Y.N., K.R. Pattipati, and M.L. Curry, "Normative design of organizations to solve a complex mission : theory and algorithms", Proceedings of the 1997 Command and Control Research and Technology Symposium, Washington, DC, 1997.

[Y. Levchuk et al, 1998] Levchuk, Y.N., Pattipati K. R., and Kleinman, D. L., "Designing adaptive organizations to process a complex mission: algorithms and applications", Proceedings of the 1998 Command \& Control Research \& Technology Symposium, NPS, Monterey, CA, 1998.

[Levebvre et al., 1997] Levebvre, E., L.A. Levebvre, and L. Prefontaine, "Technological learning and organizational context: fit and performance in SMEs", Proceedings of 30-th Hawaii International Conference on System Sciences, Vol. 3, 464-474, 1997.

[Lee and Carley, 1997] Lee, J.S. and K.M. Carley, "Adaptive strategy for improving C2 performance", Proc. 1997 Com. and Contr. Res. and Tech. Symposium, Washington, DC, USA, 1997.

[Mackenzie, 1986] Mackenzie, K.D. Organizational Design: The Organizational Audit and Analysis Technology. Alex Pub. Co., Norwood, New Jersey, 1986.

[Mackenzie et al., 1996] Mackenzie, C.F., N.J. Jefferies, W.A. Hunter, W.N. Bernhard, Y. Xiao, and R. Horst, "Comparison of self reporting of deficiencies in airway management with video analysis of actual performance", Human Factors, 38(4), pp. 623-635, 1996.

[Mathur and Pattipati, 1997] Mathur, A. and K.R. Pattipati, "Single and multi-objective optimization techniques for robust parameter design," invited paper Sadhana, Indian Academy of Sciences, 1997.

[Pachucki, 1995] Pachucki, D.E., "Environmental stress testing experiment using the Taguchi method", IEEE Transactions on Components, Packaging, and Manufacturing Technology, Part A, [see also IEEE Transactions on Components, Hybrids, and Manufacturing Technology], Vol. 18, No. 1, 3-9, 1995.

[Peddie et al., 1991] Peddie, R.A., J.H. Banks, and T.J. Haslam, "Electricity privatisation-IT's role in facilitating rapid organisational change", Engineering Management Journal, Vol. 1, No. 4 , $178-186,1991$.

[Piramuthu et al., 1994] Piramuthu, S., N. Raman, and M.J. Shaw, "Learning-based scheduling in a flexible manufacturing flow line", IEEE Transactions on Engineering Management, Vol. 41, No. 2, 172-182, 1994.

[Roberts, 1989] Roberts K., "New challenges to organizational research: high reliability organizations", Industrial crisis quarterly, Vol. 3, No. 3, 111-125, 1989.

[Sanchez, 2000] Sanchez, S.M., "Robust design: seeking the best of all possible worlds", Proceedings of the 2000 Winter Simulation Conference, 69-76, 2000. 
[Scibilia et al., 1999] Scibilia, B., A. Kobi, R. Chassagnon, and A. Barreau, "An application of the quality engineering approach reconsidered", Proceedings of the IEEE International Conference on Systems, Man, and Cybernetics. Vol. 1, 943 -947, 1999.

[Song et al., 1995] Song, A., A. Mathur, and K.R. Pattipati, " Design of process parameters using robust design techniques and multiple criteria optimization," IEEE Trans. on Systems, Man, and Cybernetics, Vol. 25, No. 11, 1437-1446, 1995.

[Taguchi, 1986] Taguchi, G. Introduction to Quality Engineering. White Plains, New York: UNIPUB/Krauss International, 1986.

[Taguchi, 1987] Taguchi, G. System of Experimental Design, Volumes 1 and 2. White Plains, New York: UNIPUB/Krauss International, 1987.

[Tsourveloudis and Phillis, 1998] Tsourveloudis N. C. and Y. A. Phillis, "Manufacturing flexibility measurement: a fuzzy logic framework", IEEE Transactions on Robotics and Automation, Vol. 14, No. 4, 1998.

[Villas Boas, 1999] Villas Boas, R.C., "Robust engineering approaches to maximize results in business, cost, engineering, human, quality and system technologies", Proceedings of the Second International Conference on Intelligent Processing and Manufacturing of Material. Vol. 1, 481484, 1999.

[Wickens, 1986] Wickens, C., "The object display: principles and a review of experimental findings", Technical Report. CPL 86-6, Champaign, IL: University of Illinios Cognitive Psychophysiology Laboratory, Department of Psychology, 1986. 